\title{
Automatic Verification of Erlang-Style Concurrency
}

\author{
Emanuele D'Osualdo \\ University of Oxford \\ emanuele.dosualdo@cs.ox.ac.uk
}

\author{
Jonathan Kochems \\ University of Oxford \\ jonathan.kochems@cs.ox.ac.uk
}

\author{
C.-H. Luke Ong \\ University of Oxford \\ luke.ong@cs.ox.ac.uk
}

\begin{abstract}
This paper presents an approach to verify safety properties of Erlang-style, higher-order concurrent programs automatically. Inspired by Core Erlang, we introduce $\lambda_{\text {ACTOR, a prototypical func- }}$ tional language with pattern-matching algebraic data types, augmented with process creation and asynchronous message-passing primitives. We formalise an abstract model of $\lambda$ AстоR programs called Actor Communicating System (ACS) which has a natural interpretation as a vector addition system, for which some verification problems are decidable. We give a parametric abstract interpretation framework for $\lambda$ ACTOR and use it to build a polytime computable, flow-based, abstract semantics of $\lambda$ AстоR programs, which we then use to bootstrap the ACS construction, thus deriving a more accurate abstract model of the input program. We have constructed Soter, a tool implementation of the verification method, thereby obtaining the first fully-automatic, infinite-state model checker for a core fragment of Erlang. We find that in practice our abstraction technique is accurate enough to verify an interesting range of safety properties. Though the ACS coverability problem is EXPSPACE-complete, Soter can analyse these verification problems surprisingly efficiently.
\end{abstract}

Keywords Verification, Infinite-State Model Checking, Static Analysis, Petri Nets, Erlang

\section{Introduction}

This paper concerns the verification of concurrent programs written in Erlang. Originally designed to program fault-tolerant distributed systems at Ericsson in the late 80s, Erlang is now a widely used, open-sourced language with support for higher-order functions, concurrency, communication, distribution, on-the-fly code reloading, and multiple platforms [2,3]. Largely because of a runtime system that offers highly efficient process creation and messagepassing communication, Erlang is a natural fit for programming multicore CPUs, networked servers, parallel databases, GUIs, and monitoring, control and testing tools.

The sequential part of Erlang is a higher order, dynamically typed, call-by-value functional language with pattern-matching algebraic data types. Following the actor model [1], a concurrent Erlang computation consists of a dynamic network of processes that communicate by message passing. Every process has a unique process identifier (pid), and is equipped with an unbounded mailbox. Messages are sent asynchronously in the sense that send is nonblocking. Messages are retrieved from the mailbox, not FIFO, but First-In-First-Firable-Out (FIFFO) via pattern-matching. A process may block while waiting for a message that matches a certain pattern to arrive in its mailbox. For a quick and highly readable introduction to Erlang, see Armstrong's CACM article [2].

Challenges. Concurrent programs are hard to write. They are just as hard to verify. In the case of Erlang programs, the inherent complexity of the verification task can be seen from several diverse sources of infinity in the state space.

$(\infty 1)$ General recursion requires a (process local) call-stack.

$(\infty 2)$ Higher-order functions are first-class values; closures can be passed as parameters or returned.

$(\infty 3)$ Data domains, and hence the message space, are unbounded: functions may return, and variables may be bound to, terms of an arbitrary size.

$(\infty 4)$ An unbounded number of processes can be spawned dynamically.

$(\infty 5)$ Mailboxes have unbounded capacity.

The challenge of verifying Erlang programs is that one must reason about the asynchronous communication of an unbounded set of messages, across an unbounded set of Turing-powerful processes.

Our goal is to verify safety properties of Erlang-like programs automatically, using a combination of static analysis and infinitestate model checking. To a large extent, the key decision of which causes of infinity to model as accurately as possible and which to abstract is forced upon us: the class consisting of a fixed set of context-free (equivalently, first-order) processes, each equipped with a mailbox of size one and communicating messages from a finite set, is already Turing powerful [10]. Our strategy is thus to abstract $(\infty 1),(\infty 2)$ and $(\infty 3)$, while seeking to analyse messagepassing concurrency, assuming $(\infty 4)$ and $(\infty 5)$.

We consider programs of $\lambda$ AстоR, a prototypical functional language with actor-style concurrency. $\lambda$ AстоR is essentially Core Erlang [5] - the official intermediate representation of Erlang code, which exhibits in full the higher-order features of Erlang, with asynchronous message-passing concurrency and dynamic process creation.

With decidable infinite-state model checking in mind, we introduce Actor Communicating System (ACS), which models the interaction of an unbounded set of communicating processes. An ACS has a finite set of control states $Q$, a finite set of pid classes $P$, a finite set of messages $M$, and a finite set of transition rules. An ACS transition rule has the shape $\iota: q \stackrel{\ell}{\rightarrow} q^{\prime}$, which means that a process of pid class $\iota$ can transition from state $q$ to state $q^{\prime}$ with (possible) communication side effect $\ell$, of which there are four kinds, namely, (i) the process makes an internal transition (ii) it extracts and reads a message $m$ from its mailbox (iii) it sends a message $m$ to a process of pid class $\iota^{\prime}$ (iv) it spawns a process of pid class $\iota^{\prime}$. ACS models are infinite state: the mailbox of a process has unbounded capacity, and the number of processes in an ACS may grow arbitrarily large. However the set of pid classes is fixed, and processes of the same pid class are not distinguishable.

An ACS can be interpreted naturally as a vector addition system (VAS), or equivalently Petri net, using counter abstraction. Recall that a VAS of dimension $n$ is given by a set of $n$-long vectors of integers regarded as transition rules. A VAS defines a 
state transition graph whose states are just $n$-long vectors of nonnegative integers. There is a transition from state $\mathbf{v}$ to state $\mathbf{v}^{\prime}$ just if $\mathbf{v}^{\prime}=\mathbf{v}+\mathbf{r}$ for some transition rule $\mathbf{r}$. It is well-known that the decision problems Coverability and LTL Model Checking for VAS are EXPSPACE-complete; Reachability is decidable but its complexity is open. We consider a particular counter abstraction of ACS, called VAS semantics, which models an ACS as a VAS of dimension $|P| \times(|Q|+|M|)$, distinguishing two kinds of counters. A counter named by a pair $(\iota, q)$ counts the number of processes of pid class $\iota$ that are currently in state $q$; a counter named by $(\iota, m)$ counts the sum total of occurrences of a message $m$ currently in the mailbox of $p$, where $p$ ranges over processes of pid class $\iota$. Using this abstraction, we can conservatively decide properties of the ACS using well-known decision procedures for VAS.

Parametric, Flow-based Abstract Interpretation. The starting point of our verification pathway is the abstraction of the sources of infinity $(\infty 1),(\infty 2)$ and $(\infty 3)$. Methods such as $k$-CFA [32] can be used to abstract higher-order recursive functions to a finite-state system. Rather than 'baking in' each type of abstraction separately, we develop a general abstract interpretation framework which is parametric on a number of basic domains. In the style of Van Horn and Might [33], we devise a machine-based operational semantics of $\lambda$ AстоR that uses store-allocated continuations. The advantage of such an indirection is that it enables the construction of a machine semantics which is 'generated' from the basic domains of Time, Mailbox and Data. We show that there is a simple notion of sound abstraction of the basic domains whereby every such abstraction gives rise to a sound abstract semantics of $\lambda$ Aстов programs (Theorem 1). Further if a given sound abstraction of the basic domains is finite and the associated auxiliary operations are computable, then the derived abstract semantics is finite and computable.

Generating an Actor Communicating System. We study the abstract semantics derived from a particular 0-CFA-like abstraction of the basic domains. However we do not use it to verify properties of $\lambda$ AстоR programs directly, as it is too coarse an abstraction to be useful. Rather, we show that a sound ACS (Theorem 3) can be constructed in polynomial time by bootstrapping from the 0-CFA-like abstract semantics. Further, the dimension of the resulting ACS is polynomial in the length of the input $\lambda$ ACTOR program. The idea is that the 0-CFA-like abstract (transition) semantics constitutes a sound but rough analysis of the control-flow of the program, which takes higher-order computation into account but communicating behaviour only minimally. The bootstrap construction consists in constraining these rough transitions with guards of the form 'receive a message of this type' or 'send a message of this type' or 'spawn a process', thus resulting in a more accurate abstract model of the input $\lambda$ ACтоR program in the form of an ACS.

Evaluation. To demonstrate the feasibility of our verification method, we have constructed a prototype implementation called Soter. Our empirical results show that the abstraction framework is accurate enough to verify an interesting range of safety properties of non-trivial Erlang programs.

Outline. In Section 2 we define the syntax of $\lambda$ ACTOR and informally explain its semantics with the help of an example program. In Section 3, we introduce Actor Communicating System and its VAS semantics. In Section 4 we present a machine-based operational semantics of $\lambda$ AстоR. In Section 5 we develop a general abstract interpretation framework for $\lambda$ AсTOR programs, parametric on a number of basic domains. In Section 6, we use a particular instantiation of the abstract interpretation to bootstrap the ACS construction. In Section 7 we present the experimental results based on our tool implementation Soter, and discuss the limitations of our approach.
Notation. We write $A^{*}$ for the set of finite sequences of elements of the set $A$, and $\epsilon$ for the null sequence. Let $a \in A$ and $l, l^{\prime} \in$ $A^{*}$, we overload '.' so that it means insertion at the top $a \cdot l$, at the bottom $l \cdot a$ or concatenation $l \cdot l^{\prime}$. We write $l_{i}$ for the $i$-th element of $l$. The set of finite partial functions from $A$ to $B$ is denoted $A \rightarrow B$. Given $f: A \rightarrow B$ we define $f[a \mapsto b]:=$ $(\lambda x$. if $(x=a)$ then $b$ else $f(x))$ and write [] for the everywhere undefined function.

\section{A Prototypical Fragment of Erlang}

In this section we introduce $\lambda$ Actor, a prototypical untyped functional language with actor concurrency. $\lambda$ AстоR is essentially single-node Core Erlang [5] - the official intermediate representation of Erlang code-without built-in functions and fault-tolerant features. It exhibits in full the higher-order features of Erlang, with message-passing concurrency and dynamic process creation.

Syntax The syntax of $\lambda$ Actor is defined as follows:

$$
\begin{aligned}
& e \in \operatorname{Exp}::=x\left|\mathrm{c}\left(e_{1}, \ldots, e_{n}\right)\right| e_{0}\left(e_{1}, \ldots, e_{n}\right) \mid \text { fun } \\
& \text { letrec } x_{1}=\text { fun }_{1} \cdot \cdots x_{n}=\text { fun }_{n} \text {. in } e \\
& \text { case } e \text { of } \text { pat }_{1} \rightarrow e_{1} ; \ldots ; \text { pat }_{n} \rightarrow e_{n} \text { end } \\
& \text { receive } p a t_{1} \rightarrow e_{1} ; \ldots ; p a t_{n} \rightarrow e_{n} \text { end } \\
& \operatorname{send}\left(e_{1}, e_{2}\right)|\operatorname{spawn}(e)| \operatorname{self}() \\
& \text { fun }::=\operatorname{fun}\left(x_{1}, \ldots, x_{n}\right) \rightarrow e \\
& \text { pat }::=x \mid c\left(\text { pat }_{1}, \ldots, \text { pat }_{n}\right)
\end{aligned}
$$

where $c$ ranges over a finite set $\Sigma$ of constructors which we consider fixed thorough out the paper.

For ease of comparison we keep the syntax close to Core Erlang and use uncurried functions, delimiters, fun and end. We write '-' for an unnamed unbound variable; using symbols from $\Sigma$, we write $n$-tuples as $\left\{e_{1}, \ldots, e_{n}\right\}$, the list constructors as cons [-| $\left.\left.\right|_{-}\right]$ and the empty list as []. Sequencing $\left(e_{1}, e_{2}\right)$ is a shorthand for $\left(\mathbf{f u n}(-) \rightarrow e_{2}\right)\left(e_{1}\right)$ and we we omit brackets for nullary constructors. The character ' $\%$ ' marks the start of a line of comment. Variable names begin with an uppercase letter, except when bound by letrec . The free variables $\mathrm{fv}(e)$ of an expression are defined as usual. A $\lambda$ ACTOR program $\mathcal{P}$ is just a closed $\lambda$ ACTOR expression.

Labels For ease of reference to program points, we associate a unique label to each sub-expression of a program. We write $\ell$ : $e$ to mean that $\ell$ is the label associated with $e$, and we often omit the label altogether. Take a term $\ell:\left(\ell_{0}: e_{0}\left(\ell_{1}: e_{1}, \ldots, \ell_{n}: e_{n}\right)\right)$, we define $\ell \cdot \arg _{i}:=\ell_{i}$ and $\operatorname{arity}(\ell):=n$.

Semantics The semantics of $\lambda$ AстоR is defined in Section 4 , but we informally present a small-step reduction semantics here to give an intuition of its model of concurrency. The rewrite rules for the cases of function application and $\lambda$-abstraction are the standard ones for call-by-value $\lambda$-calculus; we write evaluation contexts as $E[]$.

A state of the computation of a $\lambda$ ACTOR program is a set $\Pi$ of processes running in parallel. A process $\langle e\rangle_{\mathfrak{m}}^{\iota}$, identified by the pid $\iota$, evaluates an expression $e$ with mailbox $\mathfrak{m}$ holding the messages not yet consumed. Purely functional reductions with no side-effect take place in each process, independently interleaved. A spawn construct, $\operatorname{spawn}(\mathbf{f u n}() \rightarrow e)$, evaluates to a fresh pid $\iota^{\prime}$ (say), with the side-effect of the creation of a new process, $\langle e\rangle_{\epsilon}^{\iota^{\prime}}$, with pid $\iota^{\prime}$ :

$$
\langle E[\operatorname{spawn}(\operatorname{fun}() \rightarrow e)]\rangle_{\mathfrak{m}}^{\iota}\left\|\Pi \quad \longrightarrow\left\langle E\left[\iota^{\prime}\right]\right\rangle_{\mathfrak{m}}^{\iota}\right\|\langle e\rangle_{\epsilon}^{\iota^{\prime}} \| \Pi
$$

A send construct, $\operatorname{send}(\iota, v)$, evaluates to the message $v$ with the side-effect of appending it to the mailbox of the receiver process $\iota$; thus send is non-blocking:

$$
\langle E[\operatorname{send}(\iota, v)]\rangle_{\mathfrak{m}^{\prime}}^{\iota^{\prime}}\left\|\langle e\rangle_{\mathfrak{m}}^{\iota}\right\| \Pi \quad \longrightarrow\langle E[v]\rangle_{\mathfrak{m}^{\prime}}^{\iota^{\prime}}\left\|\langle e\rangle_{\mathfrak{m} \cdot v}^{\iota}\right\| \Pi
$$


The evaluation of a receive construct, receive $p_{1} \rightarrow e_{1} \ldots p_{n} \rightarrow$ $e_{n}$ end, will block if the mailbox of the process in question contains no message that matches any of the patterns $p_{i}$. Otherwise, the first message $m$ that matches a pattern, say $p_{i}$, is consumed by the process, and the computation continues with the evaluation of $e_{i}$. The pattern-matching variables in $e_{i}$ are bound by $\theta$ to the corresponding matching subterms of the message $m$; if more than one pattern matches the message, then (only) the first in textual order is fired.

$$
\begin{aligned}
&\left\langle E\left[\text { receive } p_{1} \rightarrow e_{1} \ldots p_{n} \rightarrow e_{n} \text { end }\right]\right\rangle_{\mathfrak{m} \cdot m \cdot \mathfrak{m}^{\prime}}^{\iota} \| \Pi \\
& \longrightarrow\left\langle E\left[\theta e_{i}\right]\right\rangle_{\mathfrak{m} \cdot \mathfrak{m}^{\prime}}^{\iota^{\prime}} \| \Pi
\end{aligned}
$$

Note that message passing is not First-In-First-Out but rather First-In-First-Fireable Out (FIFFO): incoming messages are queued at the end of the mailbox but the message that matches a receive construct, and is subsequently extracted, is not necessarily the first in the queue.

Example 1 (Locked Resource). Figure 1 shows an example $\lambda$ AстоR program. The code has three logical parts, which would constitute three modules in Erlang. The first part defines an Erlang behaviour $^{1}$ that governs the lock-controlled, concurrent access of a shared resource by a number of clients. A resource is viewed as a function implementing a protocol that reacts to requests; the function is called only when the lock is acquired. Note the use of higher-order arguments and return values. The function res_start creates a new process that runs an unlocked (res_free) instance of the resource. When unlocked, a resource waits for a $\{$ lock, P $\}$ message to arrive from a client $\mathrm{P}$. Upon receipt of such a message, an acknowledgement message is sent back to the client and the control is yielded to res_locked. When locked (by a client P), a resource can accept requests $\{$ req, $P, C m d\}$ from $P$-and from $P$ only-for an unspecified command $\mathrm{Cmd}$ to be executed.

After running the requested command, the resource is expected to return the updated resource handler and an answer, which may be the atom ok, which requires no additional action, or a couple \{reply, Ans which signals that the answer Ans should be sent back to the client. When an unlock message is received from $P$ the control is given back to res_free. Note that the mailbox matching mechanism allows multiple locks and requests to be sent asynchronously to the mailbox of the locked resource without causing conflicts: the pattern matching in the locked state ensures that all the pending lock requests get delayed for later consumption once the resource gets unlocked. The functions res_lock, res_unlock, res_request, res_do encapsulate the locking protocol, hiding it from the user who can then use this API as if it was purely functional.

The second part implements a simple 'shared memory cell' resource that holds a natural number, which is encoded using the constructors zero and $\{$ succ, - \} , and allows a client to read its value (the command read) or overwrite it with a new one (the $\{$ write, $X\}$ command). Without locks, a shared resource with such a protocol easily leads to race conditions.

The last part defines the function inc which accesses a locked cell to increment its value. The function add_to_cell adds $M$ to the contents of the cell by spawning $M$ processes incrementing it concurrently. Finally the entry-point of the program sets up a process with a shared locked cell and then calls add_to_cell. Note that $\mathrm{N}$ is a free variable; to make the example a program we can either close it by setting $N$ to a constant or make it range over all natural numbers with the extension described in Section 5.

\footnotetext{
${ }^{1}$ I.e. a module implementing a general purpose protocol, parametrised over another module containing the code specific to a particular instance.
}

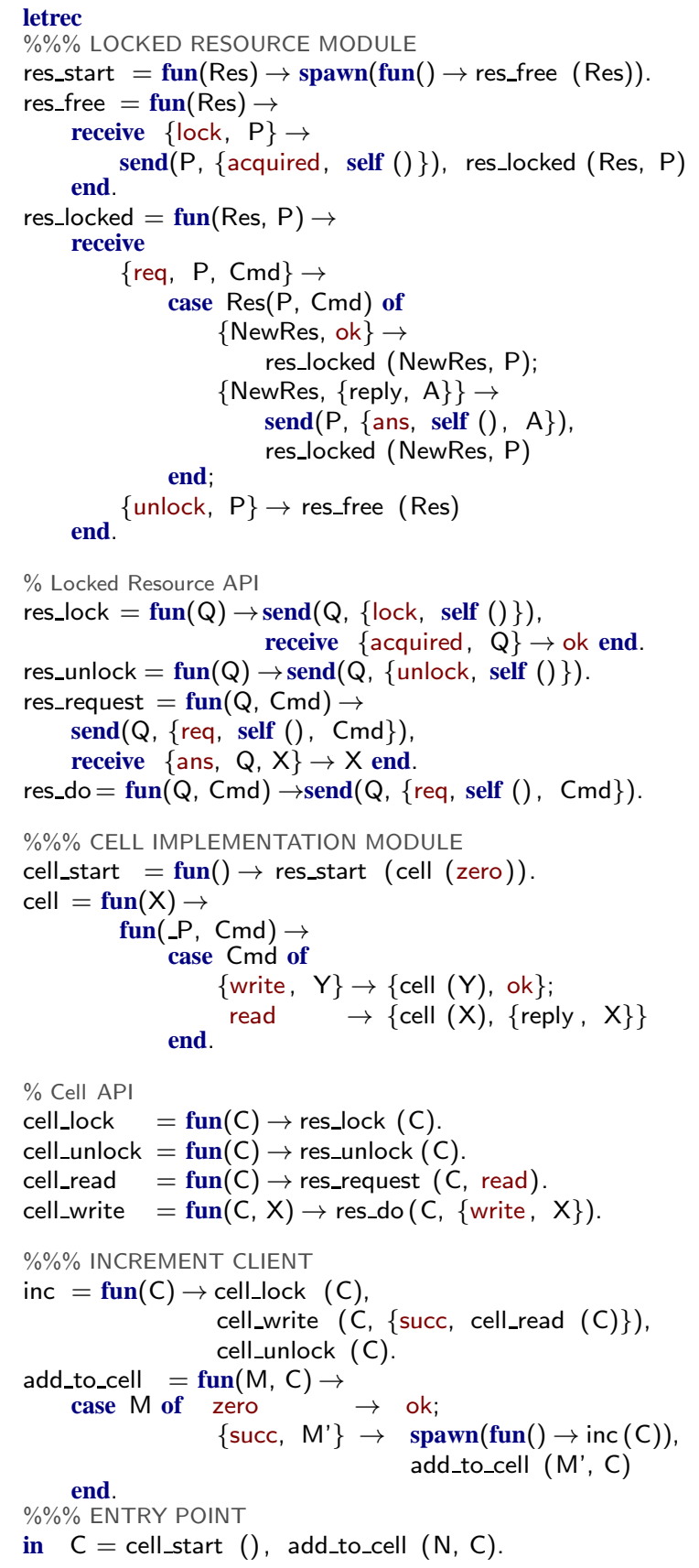

Figure 1. Locked Resource (running example)

An interesting correctness property of this code is the mutual exclusion of the lock-protected region (i.e. line 47) of the concurrent instances of inc.

Remark 1. The following Core Erlang features are not captured by $\lambda$ Aстов. (i) Module system, exception handling, arithmetic primitives, built-in data types and I/O can be straightforwardly translated or integrated into our framework. They are not treated here because they are tied to the inner workings of the Erlang runtime system. (ii) Timeouts in receives, registered processes and type guards can be supported using suitable abstractions. (iii) A proper treat- 
ment of monitor / link primitives and the multi-node semantics will require a major extension of the concrete (and abstract) semantics.

\section{Actor Communicating Systems}

In this section we explore the design space of abstract models of Erlang-style concurrency. We seek a model of computation that should capture the core concurrency and asynchronous communication features of $\lambda$ АстоR and yet enjoys the decidability of interesting verification problems. In the presence of pattern-matching algebraic data types, the (sequential) functional fragment of $\lambda$ AстоR is already Turing powerful [28]. Restricting it to a pushdown (equivalently, first-order) fragment but allowing concurrent execution would enable, using very primitive synchronization, the simulation of a Turing-powerful finite automaton with two stacks. A single finite-control process equipped with a mailbox (required for asynchronous communication) can encode a Turing-powerful queue automaton in the sense of Minsky. Thus constrained, we opt for a model of concurrent computation that has finite control, a finite number of messages, and a finite number of process classes.

Definition 1. An Actor Communicating System (ACS) $\mathcal{A}$ is a tuple $\left\langle P, Q, M, R, \iota_{0}, q_{0}\right\rangle$ where $P$ is a finite set of pid-classes, $Q$ is a finite set of control-states, $M$ is a finite set of messages, $\iota_{0} \in P$ is the pid-class of the initial process, $q_{0} \in Q$ is the initial state of the initial process, and $R$ is a finite set of rules of the form $\iota: q \stackrel{\ell}{\rightarrow} q^{\prime}$ where $\iota \in P, q, q^{\prime} \in Q$ and $\ell$ is a label that can take one of four possible forms:

- $\tau$, which represents an internal (sequential) transition of a process of pid-class $\iota$

- ? $m$ with $m \in M$ : a process of pid-class $\iota$ extracts (and reads) a message $m$ from its mailbox

- $\iota^{\prime} ! m$ with $\iota^{\prime} \in P, m \in M$ : a process of pid-class $\iota$ sends a message $m$ to a process of pid-class $\iota^{\prime}$

- $\nu \iota^{\prime} \cdot q^{\prime \prime}$ with $\iota^{\prime} \in P$ and $q^{\prime \prime} \in Q$ : a process of pid-class $\iota$ spawns a new process of pid-class $\iota^{\prime}$ that starts executing from $q^{\prime \prime}$

Now we have to give ACS a semantics, but interpreting the ACS mailboxes as FIFFO queues would yield a Turing-powerful model. Our solution is to apply a counter abstraction on mailboxes: disregard the ordering of messages, but track the number of occurrences of every message in a mailbox. Since we bound the number of pid-classes, but wish to model dynamic (and hence unbounded) spawning of processes, we apply a second counter abstraction on the control states of each pid-class: we count, for each control-state of each pid-class, the number of processes in that pid-class that are currently in that state.

It is important to make sure that such an abstraction contains all the behaviours of the semantics that uses FIFFO mailboxes: if there is a term in the mailbox that matches a pattern, then the corresponding branch is non-deterministically fired. To see the difference, take the ACS that has one process (named $\iota$ ), three control states $q, q_{1}$ and $q_{2}$, and two rules $\iota: q \stackrel{? a}{\longrightarrow} q_{1}, \iota: q \stackrel{? b}{\longrightarrow} q_{2}$. When equipped with a FIFFO mailbox containing the sequence $c a b$, the process can only evolve from $q$ to $q_{1}$ by consuming $a$ from the mailbox, since it can skip $c$ but will find a matching message (and thus not look further into the mailbox) before reaching the message $b$. In contrast, the VAS semantics would let $q$ evolve nondeterministically to both $q_{1}$ and $q_{2}$, consuming $a$ or $b$ respectively: the mailbox is abstracted to $[a \mapsto 1, b \mapsto 1, c \mapsto 1]$ with no information on whether $a$ or $b$ arrived first. However, the abstracted semantics does contain the traces of the FIFFO semantics.

The VAS semantics of an ACS is a state transition system equipped with counters (with values in $\mathbb{N}$ ) that support increment and decrement (when non-zero) operations. Such infinite-state sys- tems are known as vector addition systems (VAS), which are equivalent to Petri nets.

Definition 2 (Vector Addition System). (i) A vector addition system (VAS) $\mathcal{V}$ is a pair $(I, R)$ where $I$ is a finite set of indices (called the places of the VAS) and $R \subseteq \mathbb{Z}^{I}$ is a finite set of rules. Thus a rule is just a vector of integers of dimension $|I|$, whose components are indexed (i.e. named) by the elements of $I$.

(ii) The state transition system $\llbracket \mathcal{V} \rrbracket$ induced by a $\operatorname{VAS} \mathcal{V}=(I, R)$ has state-set $\mathbb{N}^{I}$ and transition relation

$$
\left\{(\mathbf{v}, \mathbf{v}+\mathbf{r}) \mid \mathbf{v} \in \mathbb{N}^{I}, \mathbf{r} \in R, \mathbf{v}+\mathbf{r} \in \mathbb{N}^{I}\right\}
$$

We write $\mathbf{v} \leq \mathbf{v}^{\prime}$ just if for all $i$ in $I, \mathbf{v}(i) \leq \mathbf{v}^{\prime}(i)$.

The semantics of an ACS can now be given easily in terms of a corresponding underlying vector addition system:

Definition 3 (VAS semantics). The semantics of an ACS $\mathcal{A}=$ $\left(P, Q, M, R, \iota_{0}, q_{0}\right)$ is the transition system induced by the VAS $\mathcal{V}=(I, \mathbf{R})$ where $I=P \times(Q \uplus M)$ and $\mathbf{R}=\{\mathbf{r} \mid r \in R\})$. The transformation $r \mapsto \mathbf{r}$ is defined as follows. ${ }^{2}$

\begin{tabular}{ll}
\hline ACS Rules: $r$ & \multicolumn{1}{c}{ VAS Rules: $\mathbf{r}$} \\
\hline$\iota: q \stackrel{\tau}{\rightarrow} q^{\prime}$ & {$\left[(\iota, q) \mapsto-1,\left(\iota, q^{\prime}\right) \mapsto 1\right]$} \\
$\iota: q \stackrel{? m}{\longrightarrow} q^{\prime}$ & {$\left[(\iota, q) \mapsto-1,\left(\iota, q^{\prime}\right) \mapsto 1,(\iota, m) \mapsto-1\right]$} \\
$\iota: q \stackrel{\iota^{\prime} ! m}{\longrightarrow} q^{\prime}$ & {$\left[(\iota, q) \mapsto-1,\left(\iota, q^{\prime}\right) \mapsto 1,\left(\iota^{\prime}, m\right) \mapsto 1\right]$} \\
$\iota: q \stackrel{\nu \iota^{\prime} \cdot q^{\prime \prime}}{\longrightarrow} q^{\prime}$ & {$\left[(\iota, q) \mapsto-1,\left(\iota, q^{\prime}\right) \mapsto 1,\left(\iota^{\prime}, q^{\prime \prime}\right) \mapsto 1\right]$} \\
\hline
\end{tabular}

Given a $\llbracket \mathcal{V} \rrbracket$-state $\mathbf{v} \in \mathbb{N}^{I}$, the component $\mathbf{v}(\iota, q)$ counts the number of processes in the pid-class $\iota$ currently in state $q$, while the component $\mathbf{v}(\iota, m)$ is the sum of the number of occurrences of the message $m$ in the mailboxes of the processes of the pid-class $\iota$.

While infinite-state, many non-trivial properties are decidable on VAS including reachability, coverability and place boundedness; for more details see [13]. In this paper we focus on coverability, which is EXPSPACE-complete [30]: given two states $s$ and $t$, is it possible to reach from $s$ a state $t^{\prime}$ that covers $t$ (i.e. $\left.t^{\prime} \leq t\right)$ ?

Which kinds of correctness properties of $\lambda$ AстоR programs can one specify by coverability of an ACS? We will be using ACS to over-approximate the semantics of a $\lambda$ ACTOR program, so if a state of the ACS is not coverable, then it is not reachable in any execution of the program. It follows that we can use coverability to express safety properties such as: (i) unreachability of error program locations (ii) mutual exclusion (iii) boundedness of mailboxes: is it possible to reach a state where the mailbox of pid-class $\iota$ has more than $k$ messages? If not we can allocate just $k$ memory cells for that mailbox.

\section{An Operational Semantics for $\lambda_{\mathrm{ActoR}}$}

In this section, we define an operational semantics for $\lambda$ AстоR using a time-stamped CESK* machine, following a methodology advocated by Van Horn and Might [33]. An unusual feature of such machines are store-allocated continuations which allow the recursion in a programs's control flow and data structure to be separated from the recursive structure in its state space. As we shall illustrate in Section 5, such a formalism is key to a transparently sound and parametric abstract interpretation.

A Concrete Machine Semantics. Without loss of generality, we assume that in a $\lambda$ АстоR program, variables are distinct, and constructors and cases are only applied to (bound) variables. The

\footnotetext{
${ }^{2}$ All unspecified components of the vectors $\mathbf{r}$ as defined in the table are set to zero.
} 
$\lambda$ Actor machine defines a transition system on (global) states, which are elements of the set State

$$
\begin{aligned}
s \in \text { State } & :=\text { Procs } \times \text { Mailboxes } \times \text { Store } \\
\pi \in \text { Procs } & :=\text { Pid } \rightarrow \text { ProcState } \\
\mu \in \text { Mailboxes } & :=\text { Pid } \rightarrow \text { Mailbox }
\end{aligned}
$$

An element of Procs associates a process with its (local) state, and an element of Mailboxes associates a process with its mailbox. We split the Store into two partitions

$$
\sigma \in \text { Store }:=(\text { VAddr } \rightarrow \text { Value }) \times(\text { KAddr } \rightarrow \text { Kont })
$$

each with its address space, to separate values and continuations. By abuse of notation $\sigma(x)$ shall mean the application of the first component when $x \in V A d d r$ and of the second when $x \in K A d d r$.

The local state of a process

$$
q \in \text { ProcState }:=(\text { ProgLoc } \uplus \text { Pid }) \times \text { Env } \times \text { KAddr } \times \text { Time }
$$

is a tuple, consisting of (i) a pid, or a program location ${ }^{3}$ which is a subterm of the program, labelled with its occurrence; whenever it is clear from the context, we shall omit the label; (ii) an environment, which is a map from variables to pointers to values $\rho \in E n v:=V a r \rightarrow V A d d r$; (iii) a pointer to a continuation, which indicates what to evaluate next when the current evaluation returns a value; (iv) a time-stamp, which will be described later.

Values are either closures or pids:

$$
d \in \text { Value }:=\text { Closure } \uplus \text { Pid } \quad \text { Closure }:=\text { ProgLoc } \times \text { Env }
$$

Note that, as defined, closures include both functions (which is standard) as well as constructor terms.

All the domains we define are naturally partially ordered: ProgLoc and Var are discrete partial orders, all the others are defined by the appropriate pointwise extensions.

Mailbox and Message Passing A mailbox is just a finite sequence of values: $\mathfrak{m} \in$ Mailbox $:=$ Value ${ }^{*}$. We denote the empty mailbox by $\epsilon$. A mailbox is supported by two operations:

$$
\begin{gathered}
\text { mmatch: } \text { pat }^{*} \times \text { Mailbox } \times \text { Env } \times \text { Store } \rightarrow \\
(\mathbb{N} \times(\text { Var } \rightarrow \text { Value }) \times \text { Mailbox })_{\perp} \\
\text { enq: Value } \times \text { Mailbox } \rightarrow \text { Mailbox }
\end{gathered}
$$

The function mmatch takes a list of patterns, a mailbox, the current environment and a store (for resolving pointers in the values stored in the mailbox) and returns the index of the matching pattern, a substitution witnessing the match, and the mailbox resulting from the extraction of the matched message. To model Erlang-style FIFFO mailboxes we set enq $(d, \mathfrak{m}):=\mathfrak{m} \cdot d$ and define:

$$
\operatorname{mmatch}\left(p_{1} \ldots p_{n}, \mathfrak{m}, \rho, \sigma\right):=\left(i, \theta, \mathfrak{m}_{1} \cdot \mathfrak{m}_{2}\right)
$$

such that

$$
\begin{array}{lrl}
\mathfrak{m}=\mathfrak{m}_{1} \cdot d \cdot \mathfrak{m}_{2} & \forall d^{\prime} \in \mathfrak{m}_{1} \cdot \forall j \cdot \operatorname{match}_{\rho, \sigma}\left(p_{j}, d^{\prime}\right) & =\perp \\
\theta=\operatorname{match}_{\rho, \sigma}\left(p_{i}, d\right) & \forall j<i \cdot \operatorname{match}_{\rho, \sigma}\left(p_{j}, d\right)=\perp
\end{array}
$$

where $\operatorname{match}_{\rho, \sigma}(p, d)$ seeks to match the term $d$ against the pattern $p$, following the pointers $\rho$ to the store $\sigma$ if necessary, and returning the witnessing substitution if matchable, and $\perp$ otherwise.

Evaluation Contexts as Continuations. Next we represent (in an inside-out manner) evaluation contexts as continuations. A continuation consists of a tag indicating the shape of the evaluation context, a pointer to a continuation representing the enclosing evaluation context, and, in some cases, a program location and an environment. Thus $\kappa \in$ Kont consists of the following constructs:

\footnotetext{
${ }^{3}$ Precisely a program location is a node in the abstract syntax tree of the program being analysed.
}

- Stop represents the empty context.

- $\operatorname{Arg}_{i}\left\langle\ell, v_{0} \ldots v_{i-1}, \rho, a\right\rangle$ represents the context

$$
E\left[v_{0}\left(v_{1}, \ldots, v_{i-1},[], e_{i+1}^{\prime}, \ldots, e_{n}^{\prime}\right)\right]
$$

where $e_{0}\left(e_{1}, \ldots, e_{n}\right)$ is the subterm located at $\ell ; \rho$ closes the terms $e_{i+1}, \ldots, e_{n}$ to $e_{i+1}^{\prime}, \ldots, e_{n}^{\prime}$ respectively; the address $a$ points to the continuation representing the enclosing evaluation context $E$.

Addresses, Pids and Time-Stamps. While the machine supports arbitrary concrete representations of time-stamps, addresses and pids, we present here an instance based on contours [32] which shall serve as the reference semantics of $\lambda$ AcTOR, and the basis for the abstraction of Section 5 .

A way to represent a dynamic occurrence of a symbol is the history of the computation at the point of its creation. We record history as contours which are strings of program locations

$$
t \in \text { Time }:=\text { ProgLoc* }
$$

The initial contour is just the empty sequence $t_{0}:=\epsilon$, while the tick function updates the contour of the process in question by prepending the current program location, which is always a function call (see rule Apply):

$$
\text { tick: ProgLoc } \times \text { Time } \rightarrow \text { Time } \operatorname{tick}(\ell, t):=\ell \cdot t
$$

Addresses for values $(b \in V A d d r)$ are represented by tuples comprising the current pid, the variable in question, the bound value and the current time stamp. Addresses for continuations ( $a, c \in K A d d r)$ are represented by tuples comprising the current pid, program location, environment and time (i.e. contour); or * which is the address of the initial continuation (Stop).

$$
\begin{aligned}
V A d d r & :=\text { Pid } \times \text { Var } \times \text { Data } \times \text { Time } \\
K A d d r & :=(\text { Pid } \times \text { ProgLoc } \times \text { Env } \times \text { Time }) \uplus\{*\}
\end{aligned}
$$

The data domain $(\delta \in D a t a)$ is the set of closed $\lambda$ Actor terms; the function res: Store $\times$ Value $\rightarrow$ Data resolves all the pointers of a value through the store $\sigma$, returning the corresponding closed term:

$$
\begin{aligned}
\operatorname{res}(\sigma, \iota) & :=\iota \\
\operatorname{res}(\sigma,(e, \rho)) & :=e[x \mapsto \operatorname{res}(\sigma, \sigma(\rho(x))) \mid x \in \mathrm{fv}(e)]
\end{aligned}
$$

New addresses are allocated by extracting the relevant components from the context at that point:

$$
\begin{aligned}
& \text { new }_{\mathrm{kpush}}: \text { Pid } \times \text { ProcState } \rightarrow \text { KAddr } \\
& \text { new }_{\mathrm{kpush}}(\iota,\langle\ell, \rho,-, t\rangle):=\left(\iota, \ell \cdot \arg _{0}, \rho, t\right) \\
& \text { new }_{\mathrm{kpop}}: \text { Pid } \times \text { Kont } \times \text { ProcState } \rightarrow \text { KAddr } \\
& \text { new }_{\mathrm{kpop}}\left(\iota, \kappa,\left\langle_{-},,_{-}, t\right\rangle\right):=\left(\iota, \ell \cdot \arg _{i+1}, \rho, t\right) \\
& \text { where } \kappa=\operatorname{Arg}_{i}\left\langle\ell, \ldots, \rho,_{-}\right\rangle \\
& \text {new }_{\mathrm{va}}: \text { Pid } \times \operatorname{Var} \times \text { Data } \times \text { ProcState } \rightarrow \text { VAddr } \\
& \text { new }_{\mathrm{va}}\left(\iota, x, \delta,\left\langle_{-},,_{-}, t\right\rangle\right):=(\iota, x, \delta, t)
\end{aligned}
$$

Remark 2. To enable data abstraction in our framework, the address of a value contains the data to which the variable is bound: by making appropriate use of the embedded information in the abstract semantics, we can fine-tune the data-sensitivity of our analysis, as we shall illustrate in Section 5. However when no data abstraction is intended, this data component can safely be discarded.

Following the same scheme, pids $(\iota \in$ Pid) can be identified with the contour of the spawn that generated them: Pid := $($ ProgLoc $\times$ Time $)$. Thus the generation of a new pid is defined as

$$
\begin{aligned}
& \text { new }_{\text {pid }}: \text { Pid } \times \text { ProgLoc } \times \text { Time } \rightarrow \text { Pid } \\
& \text { new }_{\text {pid }}\left(\left(\ell^{\prime}, t^{\prime}\right), \ell, t\right):=\left(\ell, \operatorname{tick}^{*}\left(t, \operatorname{tick}\left(\ell^{\prime}, t^{\prime}\right)\right)\right.
\end{aligned}
$$

where tick ${ }^{*}$ is just the simple extension of tick that prepends a whole sequence to another. Note that the new pid contains the pid 
that created it as a sub-sequence: it is indeed part of its history (dynamic context). The pid $\iota_{0}:=\left(\ell_{0}, \epsilon\right)$ is the pid associated with the starting process, where $\ell_{0}$ is just the root of the program.

Remark 3. (i) Note that the only sources of infinity for the state space are time, mailboxes and the data component of value addresses. If these domains are finite then the state space is finite and hence reachability is decidable. (ii) It is possible to present a more general version of the concrete machine semantics. We can reorganise the machine semantics so that components such as Time, Pid, Mailbox, KAddr and VAddr are presented as parameters (which may be instantiated as the situation requires). In this paper we present a contour-based machine, which is general enough to illustrate our method of verification.

Definition 4 (Concrete Semantics). Now that the state space is set up, we define a (non-deterministic) transition relation on states $(\rightarrow) \subseteq$ State $\times$ State. In Figure 2 we present the rules for application, message passing and process creation; we omit the other rules (letrec, case and treatment of pids as returned value) since they follow the same shape. The transition $s \rightarrow s^{\prime}$ is defined by a case analysis of the shape of $s$.

The rules for the purely functional reductions are a simple lifting of the corresponding rules for the sequential CESK* machine: when the currently selected process is evaluating a variable Vars its address is looked up in the environment and the corresponding value is fetched from the store and returned. Apply: When evaluating an application, control is given to each argument-including the function to be applied-in turn; FunEval and ArgEval are then applied, collecting the values in the continuation. After all arguments have been evaluated, new values are recorded in the environment (and the store), and control is given to the body of the function to be applied. The rule Receive can only fire if mmatch returns a valid match from the mailbox of the process. In case there is a match, control is passed to the expression in the matching clause, and the substitution $\theta$ witnessing the match is used to generate the bindings for the variables of the pattern. When applying a send Send, the recipient's pid is first extracted from the continuation, and enq is then called to dispatch the evaluated message to the designated mailbox. When applying a spawn Spawn, the argument must be an evaluated nullary function; a new process with a fresh pid is then created whose code is the body of the function.

One can easily add rules for run-time errors such as wrong arity in function application, non-exhaustive patterns in cases, sending to a non-pid and spawning a non-function.

\section{Parametric Abstract Interpretation}

We aim to abstract the concrete operational semantics of Section 4 isolating the least set of domains that need to be made finite in order for the abstraction to be decidable. We then state the conditions on these abstract domains that are sufficient for soundness.

In Remark 3 we identify Time, Mailbox and Data as responsible for the unboundedness of the state space. Our abstract semantics is thus parametric on the abstraction of these basic domains.

Definition 5 (Basic domains abstraction). (i) A data abstraction is a triple $\mathcal{D}=\left\langle\widehat{D a t a}, \alpha_{\mathrm{d}}, \widehat{\text { res }}\right\rangle$ where $\widehat{D a t a}$ is a flat (i.e. discretely ordered) domain of abstract data values, $\alpha_{\mathrm{d}}:$ Data $\rightarrow \widehat{\text { Data }}$ and $\widehat{\text { res }: ~} \widehat{\text { Store }} \times \widehat{\text { Value }} \rightarrow \mathscr{P}(\widehat{\text { Data }})$.

(ii) A time abstraction is a tuple $\mathcal{T}=\left\langle\widehat{\text { Time }}, \alpha_{\mathrm{t}}, \widehat{\mathrm{tick}}, \widehat{t}_{0}\right\rangle$ where $\widehat{\text { Time }}$ is a flat domain of abstract contours, $\alpha_{\mathrm{t}}$ : Time $\rightarrow \widehat{\text { Time }}$, $\widehat{t}_{0} \in \widehat{\text { Time }}$, and $\widehat{\text { tick }}:$ ProgLoc $\times \widehat{\text { Time }} \rightarrow \widehat{\text { Time }}$.

(iii) A mailbox abstraction is a tuple $\mathcal{M}=\left\langle\widehat{\text { Mailbo } x}, \leq_{\mathrm{m}}\right.$, $\left.\sqcup_{\mathrm{m}}, \alpha_{\mathrm{m}}, \widehat{\text { enq }}, \widehat{\epsilon}, \widehat{\text { mmatch }}\right\rangle$ where $\left(\widehat{\text { Mailbo } x}, \leq_{\mathrm{m}}, \sqcup_{\mathrm{m}}\right)$ is a join- semilattice with least element $\widehat{\epsilon} \in \widehat{\text { Mailbo } x}, \alpha_{\mathrm{m}}:$ Mailbox $\rightarrow$ $\widehat{\text { Mailbo } x}$ and $\widehat{\text { enq }}: \widehat{\text { Value }} \times \widehat{\text { Mailbo }} \rightarrow \widehat{\text { Mailbo } x}$ are monotone in mailboxes.

$$
\begin{aligned}
\text { mmatch : } \text { pat }^{*} \times \widehat{\text { Mailbo } x} \times \widehat{\text { Env }} \times \widehat{\text { Store }} \rightarrow \\
\qquad \mathscr{P}(\mathbb{N} \times(\text { Var } \rightarrow \widehat{\text { Value }}) \times \widehat{\text { Mailbo } x})
\end{aligned}
$$

(iv) A basic domains abstraction is a triple $\mathcal{I}=\langle\mathcal{D}, \mathcal{T}, \mathcal{M}\rangle$ consisting of a data, a time and a mailbox abstraction.

An abstract interpretation of the basic domains determines an interpretation of the other abstract domains as follows.

$$
\begin{aligned}
& \widehat{\text { State }}:=\widehat{\text { Procs }} \times \text { Mailboxes } \times \widehat{\text { Store }} \\
& \widehat{\text { Procs }}:=\widehat{\text { Pid }} \rightarrow \mathscr{P} \text { (ProcState }) \\
& \text { ProcState }:=(\text { ProgLoc } \uplus \widehat{\text { Pid }}) \times \widehat{E n v} \times \widehat{K A d d} r \times \widehat{\text { Time }} \\
& \widehat{\text { Store }}:=(\widehat{\text { VAddr }} \rightarrow \mathscr{P}(\widehat{\text { Value }})) \times(\widehat{\text { KAdd } r} \rightarrow \mathscr{P}(\widehat{\text { Kont }}))
\end{aligned}
$$

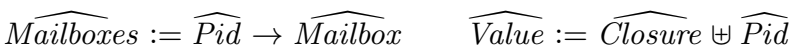

$$
\begin{aligned}
& \widehat{\text { Closure }}:=\operatorname{Prog} L o c \times \widehat{E n v} \quad \widehat{E n v}:=\operatorname{Var} \rightarrow \widehat{\operatorname{VAdd} r} \\
& \widehat{\text { Pid }}:=(\text { ProgLoc } \times \widehat{\text { Time }}) \uplus\left\{\widehat{\iota}_{0}\right\} \quad \widehat{\iota}_{0}:=\widehat{t}_{0}
\end{aligned}
$$

each equipped with an abstraction function defined by an appropriate pointwise extension. We will call all of them $\alpha$ since it will not introduce ambiguities. The abstract domain $\widehat{\text { Kont }}$ is the pointwise abstraction of Kont, and we will use the same tags as those in the concrete domain. The abstract functions nêwkpush, newkpop, $\widehat{\text { newva }}$ and $\widehat{\text { new pid }}$, are defined exactly as their concrete versions, but on the abstract domains.

When $B$ is a flat domain, the abstraction of a partial map $C=A \rightarrow B$ to $\widehat{C}=\widehat{A} \rightarrow \mathscr{P}(\widehat{B})$ is defined as

$$
\alpha_{C}(f):=\lambda \widehat{a} \in \widehat{A} .\left\{\alpha_{B}(b) \mid(a, b) \in f \text { and } \alpha_{A}(a)=\widehat{a}\right\}
$$

where the preorder on $\widehat{C}$ is $\widehat{f} \leq_{\widehat{C}} \widehat{g} \Leftrightarrow \forall \widehat{a} . \widehat{f}(\widehat{a}) \subseteq g(\widehat{a})$.

The operations on the parameter domains need to 'behave' with respect to the abstraction functions: the standard correctness conditions listed below must be satisfied by their instances. These conditions amount to requiring that what we get from an application of a concrete auxiliary function is adequately represented by the abstract result of the application of the abstract counterpart of that auxiliary function. The partial orders on the domains are standard pointwise extensions of partial orders of the parameter domains.

Definition 6 (Sound basic domains abstraction). A basic domains abstraction $\mathcal{I}$ is sound just if the following conditions are met by the auxiliary operations:

$$
\begin{gathered}
\alpha_{\mathrm{t}}(\operatorname{tick}(\ell, t)) \leq \widehat{\operatorname{tick}}\left(\ell, \alpha_{\mathrm{t}}(t)\right) \\
\widehat{\sigma} \leq \widehat{\sigma}^{\prime} \wedge \widehat{d} \leq \widehat{d} \Longrightarrow \widehat{\operatorname{res}}(\widehat{\sigma}, \widehat{d}) \leq \widehat{\operatorname{res}}\left(\widehat{\sigma}^{\prime}, \widehat{d}^{\prime}\right) \\
\forall \widehat{\sigma} \geq \alpha(\sigma) \cdot \alpha_{\mathrm{d}}(\operatorname{res}(\sigma, d)) \in \widehat{\operatorname{res}}(\widehat{\sigma}, \alpha(d)) \\
\alpha_{\mathrm{m}}(\operatorname{enq}(d, \mathfrak{m})) \leq \widehat{\operatorname{enq}}\left(\alpha(d), \alpha_{\mathrm{m}}(\mathfrak{m})\right) \quad \alpha_{\mathrm{m}}(\epsilon)=\widehat{\epsilon}
\end{gathered}
$$

if $\operatorname{mmatch}(\vec{p}, \mathfrak{m}, \rho, \sigma)=\left(i, \theta, \mathfrak{m}^{\prime}\right)$ then $\forall \widehat{\mathfrak{m}} \geq \alpha(\mathfrak{m}), \forall \widehat{\sigma} \geq$ $\alpha(\sigma), \exists \widehat{\mathfrak{m}}^{\prime} \geq \alpha\left(\mathfrak{m}^{\prime}\right)$ such that

$$
\left(i, \alpha(\theta), \widehat{\mathfrak{m}}^{\prime}\right) \in \widehat{\operatorname{mmatch}}(\vec{p}, \widehat{\mathfrak{m}}, \alpha(\rho), \widehat{\sigma})
$$

Following the Abstract Interpretation framework, one can exploit the soundness constraints to derive, by algebraic manipulation, the definitions of the abstract auxiliary functions which would then be correct by construction [24].

Definition 7 (Abstract Semantics). Once the abstract domains are fixed, the rules that define the abstract transition relation are 

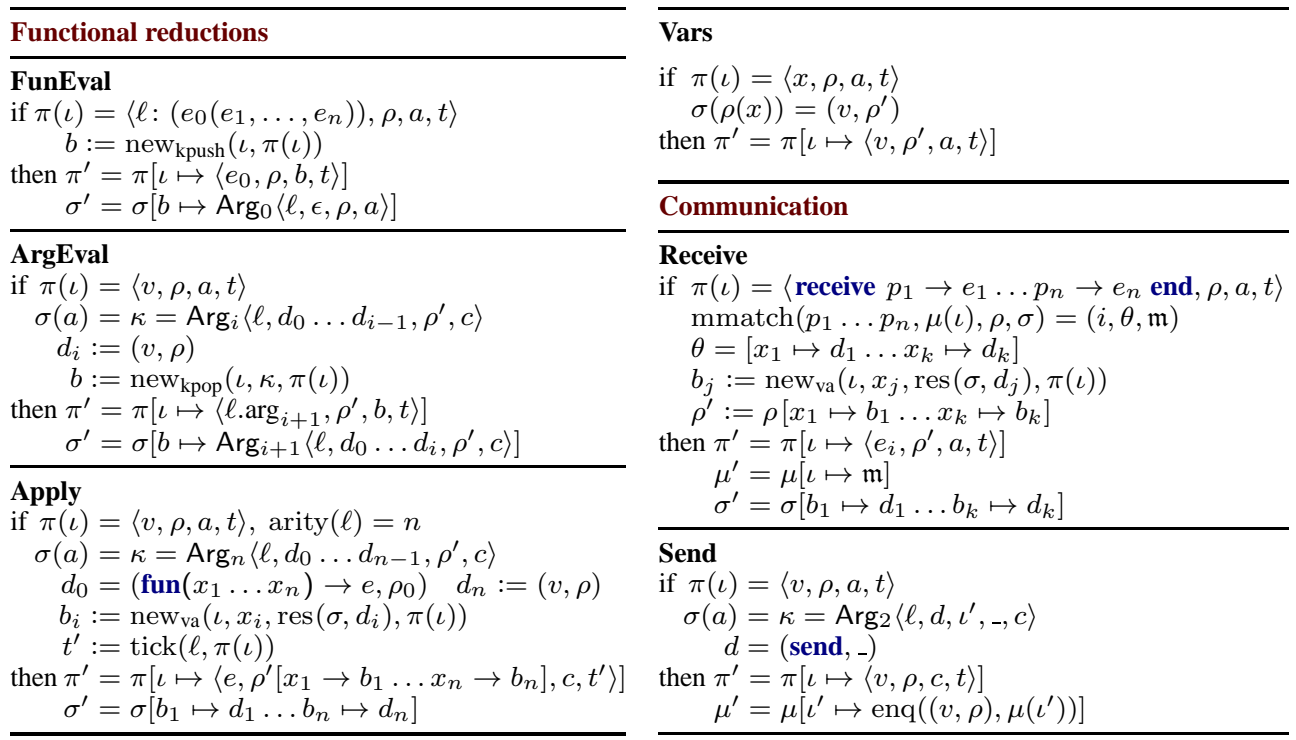

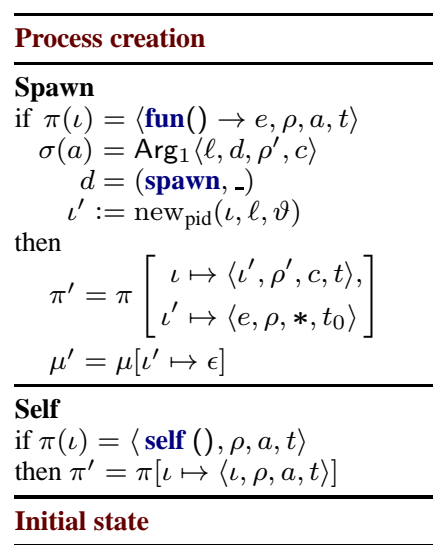

Init The initial state associated with a program $\mathcal{P}$ is $s_{\mathcal{P}}:=\left\langle\pi_{0}, \mu_{0}, \sigma_{0}\right\rangle$ where $\pi_{0}=\left[\iota_{0} \mapsto\left\langle\mathcal{P},[], *, t_{0}\right\rangle\right]$

$$
\mu_{0}=\left[\iota_{0} \mapsto \epsilon\right]
$$$$
\sigma_{0}=[* \mapsto \text { Stop }]
$$

Figure 2. Operational Semantics Rules. The tables define the transition relation $s=\langle\pi, \mu, \sigma, \vartheta\rangle \rightarrow\left\langle\pi^{\prime}, \mu^{\prime}, \sigma^{\prime}, \vartheta^{\prime}\right\rangle=s^{\prime}$ by cases; the primed components of the state are identical to the non-primed components, unless indicated otherwise in the "then" part of the rule. The meta-variable $v$ stands for terms that cannot be further rewritten such as $\lambda$-abstractions, constructor applications and un-applied primitives.

straightforward abstractions of the original ones. In Figure 3, we present the abstract counterparts of the rules for the operational semantics in Figure 2, defining the non-deterministic abstract transition relation on abstract states $(\rightsquigarrow) \subseteq \widehat{\text { State }} \times \widehat{\text { State }}$. When referring to a particular program $\mathcal{P}$, the abstract semantics is the portion of the graph reachable from $s_{\mathcal{P}}$.

Theorem 1 (Soundness of Analysis). Given a sound abstraction of the basic domains, if $s \rightarrow s^{\prime}$ and $\alpha_{c f a}(s) \leq u$, then there exists $u^{\prime} \in \widehat{\text { State }}$ such that $\alpha_{c f a}\left(s^{\prime}\right) \leq u^{\prime}$ and $u \rightsquigarrow u^{\prime}$.

See Appendix B for a proof of the Theorem.

Now that we have defined a sound abstract semantics we give sufficient conditions for its computability.

Theorem 2 (Decidability of Analysis). If a given (sound) abstraction of the basic domains is finite, then the derived abstract transition relation defined in Figure 3 is finite; it is also decidable if the associated auxiliary operations (in Definition 6) are computable.

Proof. The proof is by a simple inspection of the rules: all the individual rules are decidable and the state space is finite.

A Simple Mailbox Abstraction Abstract mailboxes need to be finite too in order for the analysis to be computable. By abstracting addresses (and data) to a finite set, values, and thus messages, become finite too. The only unbounded dimension of a mailbox becomes then the length of the sequence of messages. We then abstract mailboxes by losing information about the sequence and collecting all the incoming messages in an un-ordered set:

$$
\mathcal{M}_{\text {set }}:=\left\langle\mathscr{P}(\widehat{\text { Value }}), \subseteq, \cup, \alpha_{\text {set }}, \widehat{\text { enq }}_{\text {set }}, \emptyset, \widehat{\text { mmatch }}_{\text {set }}\right\rangle
$$

where the abstract version of enq is the insertion in the set, as easily derived from the soundness requirement; the matching function is similarly derived from the correctness condition: writing $\vec{p}=$ $p_{1} \ldots p_{n}$

$$
\begin{aligned}
& \alpha_{\text {set }}(\mathfrak{m}):=\left\{\alpha(d) \mid \exists i \cdot \mathfrak{m}_{i}=d\right\} \quad \begin{array}{l}
\widehat{\operatorname{enq}}_{\text {set }}(\widehat{d}, \widehat{\mathfrak{m}}):=\{\widehat{d}\} \cup \widehat{\mathfrak{m}} \\
\operatorname{mmatch}_{\text {set }}(\vec{p}, \widehat{\mathfrak{m}}, \widehat{\rho}, \widehat{\sigma}):=\left\{(i, \widehat{\theta}, \widehat{\mathfrak{m}}) \mid \begin{array}{l}
\widehat{d} \in \widehat{\mathfrak{m}}, \\
\widehat{\theta} \in \widehat{\operatorname{match}_{\widehat{\rho}, \widehat{\sigma}}\left(p_{i}, \widehat{d}\right)}
\end{array}\right\}
\end{array}
\end{aligned}
$$

We omit the straightforward proof that this constitutes a sound abstraction.

Abstracting Data. We included data in the value addresses in the definition of $V A d d r$, cutting contours would have been sufficient to make this domain finite. A simple solution is to discard the value completely by using the trivial data abstraction Data $_{0}:=$ $\{-\}$ which is sound. If more precision is needed, any finite dataabstraction would do: the analysis would then be able to distinguish states that differ only because of different bindings in their frame.

We present here a data abstraction particularly well-suited to languages with algebraic data-types such as $\lambda$ AстоR: the abstraction $\lfloor e\rfloor_{\widehat{\sigma}, D}$ discards every sub-term of $e$ that is nested at a deeper level than a parameter $D$.

$$
\begin{aligned}
& \lfloor(e, \widehat{\rho})\rfloor_{\widehat{\sigma}, 0}:=\{-\} \quad\lfloor(\text { fun } \ldots, \widehat{\rho})\rfloor_{\widehat{\sigma}, D+1}:=\{-\} \\
& \left\lfloor\left(c\left(x_{1} \ldots x_{n}\right), \widehat{\rho}\right)\right\rfloor_{\widehat{\sigma}, D+1}:=\left\{\begin{array}{l|l}
c\left(\widehat{\delta}_{1} \ldots \widehat{\delta}_{n}\right) & \begin{array}{l}
\widehat{d}_{i} \in \widehat{\sigma}\left(\widehat{\rho}\left(x_{i}\right)\right), \\
\widehat{\delta}_{i} \in\left\lfloor\widehat{d}_{i}\right\rfloor_{\widehat{\sigma}, D}
\end{array}
\end{array}\right\}
\end{aligned}
$$

where _ is a placeholder for discarded subterms.

An analogous D-deep abstraction can be easily defined for concrete values and we use the same notation for both; we use the notation $\lfloor\delta\rfloor_{D}$ for the analogous function on elements of Data.

We define $\mathcal{D}_{D}=\left\langle\right.$ Data $\left._{D}, \alpha_{\mathrm{D}}, \widehat{\mathrm{res}}_{D}\right\rangle$ to be the 'depth-D' data abstraction where

$$
\begin{aligned}
\operatorname{Data}_{D+1} & :=\{-\} \cup\left\{c\left(\widehat{\delta}_{1} \ldots \widehat{\delta}_{n}\right) \mid \widehat{\delta}_{i} \in \text { Data }_{D}\right\} \\
\alpha_{\mathrm{D}}(\delta) & :=\lfloor\delta\rfloor_{D} \quad \widehat{\operatorname{res}}_{D}(\widehat{\sigma}, \widehat{d}):=\lfloor\widehat{d}\rfloor_{\widehat{\sigma}, D}
\end{aligned}
$$

The proof of its soundness is easy and we omit it.

Abstracting Time. Let us now define a specific time abstraction that amounts to a concurrent version of a standard $k$-CFA. A $k$-CFA is an analysis parametric in $k$, which is able to distinguish dynamic contexts up to the bound given by $k$. We proceed as in standard $k$-CFA by truncating contours at length $k$ to obtain their abstract 


\begin{tabular}{|c|}
\hline Functional abstract reductions \\
\hline$\overline{\text { AbsFunEval }}$ \\
\hline $\begin{array}{l}\text { if } \widehat{\pi}(\widehat{\iota}) \ni \widehat{q}=\left\langle\ell:\left(e_{0}\left(e_{1}, \ldots, e_{n}\right)\right), \widehat{\rho}, \widehat{a}, \widehat{t}\right\rangle \\
\widehat{b}:=\text { newkpush }(\widehat{\iota}, \widehat{q}) \\
\text { then } \begin{aligned} & \widehat{\pi}^{\prime}=\widehat{\pi} \sqcup\left[\widehat{\iota} \mapsto\left\{\left\langle e_{0}, \widehat{\rho}, \widehat{b}, \widehat{t}\right\rangle\right\}\right] \\
& \widehat{\sigma}^{\prime}=\widehat{\sigma} \sqcup\left[\widehat{b} \mapsto\left\{\operatorname{Arg}_{0}\langle\ell, \epsilon, \widehat{\rho}, \widehat{a}\rangle\right\}\right] \\
&\end{aligned}\end{array}$ \\
\hline AbsArgEval \\
\hline $\begin{aligned} \text { if } \widehat{\pi}(\widehat{\iota}) & \ni\langle v, \widehat{\rho}, \widehat{a}, \widehat{t}\rangle \\
\widehat{\sigma}(\widehat{a}) & \ni \widehat{\kappa}=\operatorname{Arg}_{i}\left\langle\ell, \widehat{d}_{0} \ldots \widehat{d}_{i-1}, \widehat{\rho}^{\prime}, \widehat{c}\right\rangle \\
\widehat{d}_{i} & :=(v, \widehat{\rho}) \\
\widehat{b} & :=\operatorname{ne\mathrm {ew}_{\mathrm {kpop}}}(\widehat{\iota}, \widehat{\kappa}, \widehat{q}) \\
\text { then } \widehat{\pi}^{\prime} & =\widehat{\pi} \sqcup\left[\widehat{\iota} \mapsto\left\{\left\langle\ell \arg _{i+1}, \widehat{\rho}^{\prime}, \widehat{b}, \widehat{t}\right\rangle\right\}\right] \\
\widehat{\sigma}^{\prime} & =\widehat{\sigma} \sqcup\left[\widehat{b} \mapsto\left\{\operatorname{Arg}_{i+1}\left\langle\ell, \widehat{d}_{0} \ldots \widehat{d}_{i}, \widehat{\rho}^{\prime}, \widehat{c}\right\rangle\right\}\right]\end{aligned}$ \\
\hline AbsApply \\
\hline if $\begin{aligned} \widehat{\pi}(\widehat{\iota}) & \ni \widehat{q}=\langle v, \widehat{\rho}, \widehat{a}, \widehat{t}\rangle, \operatorname{arity}(\ell)=n \\
\widehat{\sigma}(\widehat{a}) & \ni \operatorname{Arg}\left\langle\ell, \widehat{d}_{0} \ldots \widehat{d}_{n-1}, \widehat{\rho}^{\prime}, \widehat{c}\right\rangle \\
\widehat{d}_{0} & =\left(\operatorname{fun}\left(x_{1} \ldots x_{n}\right) \rightarrow e, \widehat{\rho}_{0}\right) \quad \widehat{d}_{n}:=(v, \widehat{\rho}) \\
\widehat{\delta}_{i} & \in \widehat{\operatorname{res}}\left(\widehat{\sigma}, \widehat{d}_{i},\right) \\
\widehat{b}_{i} & :=\widehat{\operatorname{new}}\left(\widehat{\iota}, x_{i}, \widehat{\delta}_{i}, \widehat{q}\right) \\
\widehat{\rho}^{\prime \prime}: & =\widehat{\rho}^{\prime}\left[x_{1} \mapsto \widehat{b}_{1} \ldots x_{n} \mapsto \widehat{b}_{n}\right] \\
\text { then } \widehat{\pi}^{\prime} & =\widehat{\pi} \sqcup\left[\widehat{\iota} \mapsto\left\{\left\langle e, \widehat{\rho}^{\prime \prime}, \widehat{c}, \widehat{\operatorname{tick}}(l, \widehat{t})\right\rangle\right\}\right] \\
\widehat{\sigma}^{\prime} & =\widehat{\sigma} \sqcup\left[\widehat{b}_{1} \mapsto\left\{\widehat{d}_{1}\right\} \ldots \widehat{b}_{n} \mapsto\left\{\widehat{d}_{n}\right\}\right]\end{aligned}$ \\
\hline
\end{tabular}

AbsVars
if $\widehat{\pi}(\widehat{\iota}) \ni\langle x, \widehat{\rho}, \widehat{a}, \widehat{t}\rangle$
$\quad \widehat{\sigma}(\widehat{\rho}(x)) \ni\left(v, \widehat{\rho}^{\prime}\right)$
then $\widehat{\pi}^{\prime}=\widehat{\pi} \sqcup\left[\widehat{\iota} \mapsto\left\{\left\langle v, \widehat{\rho}^{\prime}, \widehat{a}, \widehat{t}\right\rangle\right\}\right]$

\begin{tabular}{l}
$\overline{\text { Abstract communication }}$ \\
\hline AbsReceive \\
if $\widehat{\pi}(\widehat{\iota}) \ni \widehat{q}=\langle e, \widehat{\rho}, \widehat{a}, \widehat{t}\rangle$ \\
$e=$ receive $p_{1} \rightarrow e_{1} \ldots p_{n} \rightarrow e_{n}$ end \\
mmatch $\left(p_{1} \ldots p_{n}, \widehat{\mu}(\widehat{\iota}), \widehat{\rho}, \widehat{\sigma}\right) \ni(i, \widehat{\theta}, \widehat{\mathfrak{m}})$ \\
$\widehat{\theta}=\left[x_{1} \mapsto \widehat{d}_{1} \ldots x_{k} \mapsto \widehat{d_{k}}\right]$ \\
$\widehat{\delta}_{j} \in \widehat{\operatorname{res}}\left(\widehat{\sigma}, \widehat{d}_{j}\right)$ \\
$\widehat{b}_{j}:=\widehat{\operatorname{new}}\left(\widehat{\iota}, x_{j}, \widehat{\delta}_{j}, \widehat{q}\right)$ \\
$\widehat{\rho}^{\prime}:=\widehat{\rho}\left[x_{1} \mapsto \widehat{b}_{1} \ldots x_{k} \mapsto \widehat{b}_{k}\right]$ \\
then $\widehat{\pi}^{\prime}=\widehat{\pi} \sqcup\left[\widehat{\iota} \mapsto\left\{\left\langle e_{i}, \widehat{\rho}^{\prime}, \widehat{a}, \widehat{t}\right\rangle\right\}\right]$ \\
$\widehat{\mu}^{\prime}=\widehat{\mu}[\widehat{\iota} \mapsto \widehat{\mathfrak{m}}]$ \\
$\widehat{\sigma}^{\prime}=\widehat{\sigma} \sqcup\left[\widehat{b}_{1} \mapsto\left\{\widehat{d}_{1}\right\} \ldots \widehat{b}_{k} \mapsto\left\{\widehat{d}_{k}\right\}\right]$
\end{tabular}

AbsSend

$$
\text { if } \begin{aligned}
\widehat{\pi}(\widehat{\iota}) & \ni\langle v, \widehat{\rho}, \widehat{a}, \widehat{t}\rangle \\
\widehat{\sigma}(\widehat{a}) & \ni \operatorname{Arg}_{2}\left\langle\ell, \widehat{d}, \widehat{\iota},{ }_{-}, \widehat{c}\right\rangle \\
\widehat{d} & =(\mathbf{s e n d},-) \\
\text { then } \widehat{\pi}^{\prime} & =\widehat{\pi} \sqcup[\widehat{\iota} \mapsto\{\langle v, \widehat{\rho}, \widehat{c}, \widehat{t}\rangle\}] \\
\widehat{\mu}^{\prime} & =\widehat{\mu}\left[\widehat{\iota}^{\prime} \mapsto \widehat{\operatorname{enq}}\left((v, \widehat{\rho}), \widehat{\mu}\left(\widehat{\iota}^{\prime}\right)\right)\right]
\end{aligned}
$$

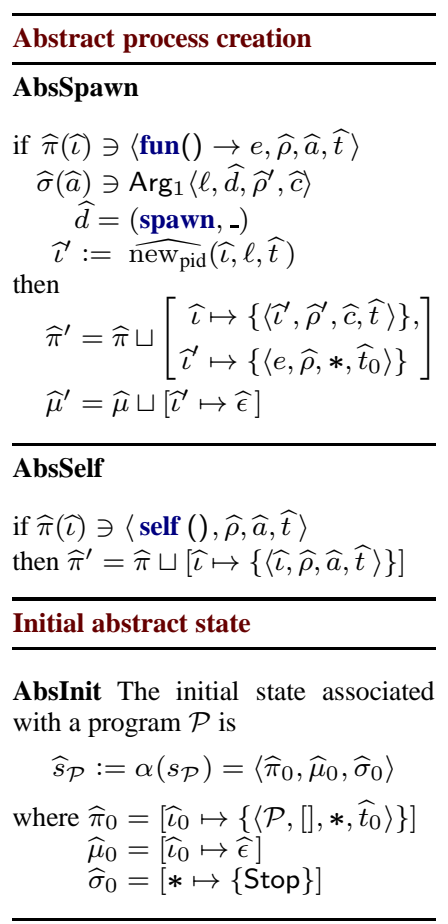

Figure 3. Rules defining the Abstract Semantics. The tables describe the conditions under which a transition $\widehat{s}=\langle\widehat{\pi}, \widehat{\mu}, \widehat{\sigma}\rangle \rightsquigarrow\left\langle\widehat{\pi}^{\prime}, \widehat{\mu}^{\prime}\right.$, $\left.\widehat{\sigma}^{\prime}\right\rangle=\widehat{s}^{\prime}$ can fire; the primed versions of the components of the states are identical to the non-primed ones unless indicated otherwise in the "then" part of the corresponding rule. We write $\sqcup$ for the join operation of the appropriate domain.

counterparts:

$$
\text { Time }_{k}:=\bigcup_{0 \leq i \leq k} \text { ProgLoc }^{i} \quad \alpha_{\mathrm{t}}^{k}\left(\ell_{1} \ldots \ell_{k} \cdot t\right):=\ell_{1} \ldots \ell_{k}
$$

The simplest analysis we can then define is the one induced by the basic domains abstraction $\left\langle\right.$ Data $_{0}$, Time $\left._{0}, \widehat{\text { Mailbo }} x_{\text {set }}\right\rangle$. With this instantiation many of the domains collapse in to singletons. Implementing the analysis as it is would lead however to an exponential algorithm because it would record separate store and mailboxes for each abstract state. To get a better complexity bound, we apply a widening following the lines of [33, Section 7]: instead of keeping a separate store and separate mailboxes for each state we can join them keeping just a global copy of each. This reduces significantly the space we need to explore: the algorithm becomes polynomial time in the size of the program (which is reflected in the size of ProgLoc).

Considering other abstractions for the basic domains easily leads to exponential algorithms; in particular, the state-space grows linearly wrt the size of abstract data so the complexity of the analysis using Data $_{D}$ is exponential in $D$.

Dealing with open programs. Often it is useful to verify an open expression where its input is taken from a regular set of terms (see [28]). We can reproduce this in our setting by introducing a new primitive choice that non-deterministically calls one of its arguments. For instance, an interesting way of closing $\mathrm{N}$ in Example 1 would be by binding it to any_num():

$$
\begin{aligned}
& \text { letrec ... } \\
& \text { any_num }()=\text { choice }(\text { fun }() \rightarrow \text { zero, } \\
& \text { fun }() \rightarrow \text { succ, any_num }()\}) \text {. } \\
& \text { in } \mathrm{C}=\text { cell_start }() \text {, add_to_cell (any_num }(), \mathrm{C}) \text {. }
\end{aligned}
$$

Now the uncoverability of the state where more than one instance of inc is running the protected section would prove that mutual exclusion is ensured for any number of concurrent copies of inc.

\section{Generating the Actor Communicating System}

The CFA algorithm we presented allows us to derive a sound 'flat' representation of the control-flow of the program. The analysis takes into account higher-order computation and (limited) information about synchronization. Now that we have this rough scheme of the possible transitions, we can 'guard' those transitions with actions which must take place in their correspondence; these guards, in the form of 'receive a message of this form' or 'send a message of this form' or 'spawn this process' cannot be modelled faithfully while retaining decidability of useful verification problems, as noted in Section 3. The best we can do, while remaining sound, is to relax the synchronization and process creation primitives with counting abstractions and use the guards to restrict the applicability of the transitions. In other words, these guarded (labelled) rules will form the definition of an ACS that simulates the semantics of the input $\lambda$ ACTOR program.

Terminology. We identify a common pattern of the rules in Figure 3. In each rule $\mathbf{R}$, the premise distinguishes an abstract pid $\widehat{\iota}$ and an abstract process state $\widehat{q}=\langle e, \widehat{\rho}, \widehat{a}, \widehat{t}\rangle$ associated with $\widehat{\iota}$ i.e. $\widehat{q} \in \widehat{\pi}(\widehat{\iota})$ and the conclusion of the rule associates a new abstract process state-call it $\widehat{q}^{\prime}$-with $\widehat{\iota}$ i.e. $\widehat{q}^{\prime} \in \widehat{\pi}^{\prime}(\widehat{\iota})$. Henceforth we shall refer to $\left(\widehat{\iota}, \widehat{q}, \widehat{q}^{\prime}\right)$ as the active components of the rule $\mathbf{R}$.

Definition 8 (Generated ACS). Given a $\lambda$ Aстов program $\mathcal{P}$, a sound basic domains abstraction $\mathcal{I}=\langle\mathcal{T}, \mathcal{M}, \mathcal{D}\rangle$ and a sound data abstraction for messages $\mathcal{D}_{\mathrm{msg}}=\left\langle\widehat{M s g}, \alpha_{\mathrm{msg}}, \widehat{\mathrm{res}_{\mathrm{msg}}}\right\rangle$

the Actor communicating system generated by $\mathcal{P}, \mathcal{I}$ and $\mathcal{D}_{\text {msg }}$ is defined as

$$
\mathcal{A}_{\mathcal{P}}:=\left\langle\widehat{\text { Pid }}, \text { ProcState }, \widehat{M s g}, R, \alpha\left(\iota_{0}\right), \alpha\left(\pi_{0}\left(\iota_{0}\right)\right)\right\rangle
$$

where $s_{\mathcal{P}}=\left\langle\pi_{0}, \mu_{0}, \sigma_{0}, t_{0}\right\rangle$ is the initial state (according to Init) with $\pi_{0}=\left[\iota_{0} \mapsto\left\langle\mathcal{P},[], *, t_{0}\right\rangle\right]$ and the rules in $R$ are defined by induction over the following rules. 
(i) If $\widehat{s} \rightsquigarrow \widehat{s}^{\prime}$ is proved by rule AbsFunEval or AbsArgEval or AbsApply with active components $\left(\widehat{\iota}, \widehat{q}, \widehat{q}^{\prime}\right)$, then

$$
\widehat{\iota}: \widehat{q} \stackrel{\tau}{\rightarrow} \widehat{q}^{\prime} \in R
$$

(ii) If $\widehat{s} \rightsquigarrow \widehat{s}$ is proved by AbsReceive with active components $\left(\widehat{\iota}, \widehat{q}, \widehat{q}^{\prime}\right)$ where $\widehat{d}=\left(p_{i}, \widehat{\rho}^{\prime}\right)$ is the abstract message matched by mmatch and $\widehat{m} \in \widehat{\text { res }}_{\mathrm{msg}}(\widehat{\sigma}, \widehat{d})$, then

$$
\widehat{\iota}: \widehat{q} \stackrel{? m}{\longrightarrow} \widehat{q}^{\prime} \in R
$$

(iii) If $\widehat{s} \rightsquigarrow \widehat{s}$ is proved by AbsSend with active components $\left(\widehat{\iota}, \widehat{q}, \widehat{q}^{\prime}\right)$ where $\widehat{d}$ is the abstract value that is sent and $\widehat{m} \in$ $\widehat{\operatorname{res}}_{\mathrm{mgg}}(\widehat{\sigma}, \widehat{d})$, then

$$
\widehat{\iota}: \widehat{q} \stackrel{\widehat{\iota}^{\prime} ! \widehat{m}}{\longrightarrow} \widehat{q}^{\prime} \in R
$$

(AcsSend)

(iv) If $\widehat{s} \rightsquigarrow \widehat{s}^{\prime}$ is proved by AbsSpawn with active component $\left(\widehat{\iota}, \widehat{q}, \widehat{q}^{\prime}\right)$ where $\widehat{\iota}$ is the new abstract pid that is generated in the premise of the rule, which gets associated with the process state $\widehat{q}^{\prime \prime}=\langle e, \widehat{\rho}, *\rangle$ then

$$
\widehat{\iota}: \widehat{q} \stackrel{\nu \widehat{\iota}^{\prime} \cdot \widehat{q}^{\prime \prime}}{\longrightarrow} \widehat{q}^{\prime} \in R
$$

As we will make precise later, keeping $\widehat{\operatorname{Pid}}$ and ProcState small is of paramount importance for the model checking of the generated ACS to be feasible. This is the main reason why we keep the message abstraction independent from the data abstraction: this allows us to increase precision with respect to types of messages, which is computationally cheap, and keep the expensive precision on data as low as possible. It is important to note that these two 'dimensions' are in fact independent and a more precise message space enhances the precision of the ACS even when using $D a t a_{0}$ as the data abstraction.

In our examples (and in our implementation) we use a Data $_{D}$ abstraction for messages where $D$ is the maximum depth of the receive patterns of the program.

Definition 9. The abstraction function

$$
\alpha_{\text {acs }}: \text { State } \rightarrow(\widehat{\text { Pid }} \times(\text { ProcState } \uplus \widehat{M s g}) \rightarrow \mathbb{N})
$$

relating concrete states and states of the ACS is defined as

$$
\alpha_{\mathrm{acs}}(s):= \begin{cases}(\widehat{\iota}, \widehat{q}) & \mapsto|\{\iota \mid \alpha(\iota)=\widehat{\iota}, \alpha(\pi(\iota))=\widehat{q}\}| \\
(\widehat{\iota}, \widehat{m}) & \mapsto\left|\left\{(\iota, i) \mid \begin{array}{l}
\alpha(\iota)=\widehat{\iota}, \\
\alpha_{\mathrm{msg}}\left(\operatorname{res}\left(\sigma, \mu(\iota)_{i}\right)\right)=\widehat{m}
\end{array}\right\}\right|\end{cases}
$$

where $s=\langle\pi, \mu, \sigma\rangle$.

It is important to note that most of the decidable properties of the generated ACS are not even expressible on the CFA graph alone: being able to predicate on the contents of the counters means we can decide boundedness, mutual exclusion and many other expressive properties. The next example shows one simple way in which the generated ACS can be more precise than the bare CFA graph.

Example 2 (Generated ACS). Given the following program:

$$
\begin{aligned}
& \text { letrec } \\
& \text { server }=\mathbf{f u n}() \rightarrow \text { receive }\{\text { init }, P, X\} \rightarrow \\
& \text { end. } \\
& \text { send }(P, \text { ok }) \text {, do_serve }(X) \\
& \text { do_serve }=\text { fun }(X) \rightarrow \text { receive } \\
& \begin{aligned}
\left\{\text { init },-{ }^{-}-\right\} & \rightarrow \text { error } ; \\
\{\text { set }, \mathrm{Y}\} \rightarrow & \text { do_serve }(\mathrm{Y}) \\
\text { get }, \mathrm{P}\} \rightarrow & \text { send }(\mathrm{P}, \mathrm{X}) \\
& \text { do_serve }(\mathrm{X})
\end{aligned}
\end{aligned}
$$

in $S=\operatorname{spawn}($ server $), \operatorname{send}(S,\{$ init, self ()$, a\})$, receive ok $\rightarrow \operatorname{send}(S$, \{set, b $\})$ end our algorithm would output the following ACS starting from 'main': ${ }^{4}$

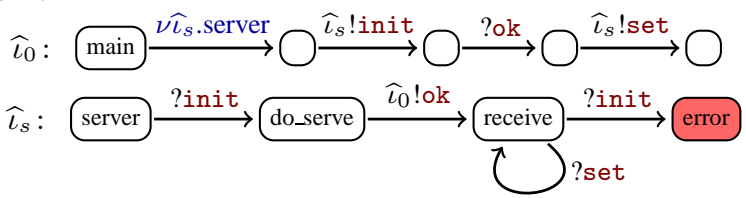

The error state is reachable in the CFA graph but not in its Parikh semantics: the token init is only sent once and never after ok is sent back to the main process. Once init has been consumed in the transition from 'server' to 'do_serve' the counter for it will remain set to zero forever.

Theorem 3 (Soundness of generated ACS). For all choices of $\mathcal{I}$ and $\mathcal{D}_{m s g}$, for all concrete states $s$ and $s^{\prime}$, if $s \rightarrow s^{\prime}$ and $\alpha_{a c s}(s) \leq \mathbf{v}$ then there exists $\mathbf{v}^{\prime}$ such that $\alpha_{a c s}\left(s^{\prime}\right) \leq \mathbf{v}^{\prime}$, and $\mathbf{v} \rightarrow$ acs $\mathbf{v}^{\prime}$.

See Appendix $\mathrm{C}$ for a proof of the Theorem.

Corollary 1 (Simulation). Let $\mathcal{A}_{\mathcal{P}}$ be the ACS derived from a given $\lambda$ Астов program $\mathcal{P}$. We have $\llbracket \mathcal{A}_{\mathcal{P}} \rrbracket$ simulates the semantics of $\mathcal{P}$ : for each $\mathcal{P}$-run $s \rightarrow s_{1} \rightarrow s_{2} \rightarrow \ldots$, there exists a $\llbracket \mathcal{A}_{\mathcal{P}} \rrbracket$-run $\mathbf{v} \rightarrow$ acs $\mathbf{v}_{1} \rightarrow$ acs $\mathbf{v}_{2} \rightarrow$ acs $\ldots$ such that $\alpha_{\text {acs }}(s)=\mathbf{v}$ and for all $i$, $\alpha_{a c s}\left(s_{i}\right) \leq \mathbf{v}_{i}$

Simulation preserves all paths so reachability (and coverability) is preserved.

Corollary 2. If there is no $\mathbf{v} \geq \alpha_{a c s}\left(s^{\prime}\right)$ such that $\alpha_{a c s}(s) \rightarrow_{\text {acs }}^{*} \mathbf{v}$ then $s \mathrm{~A}^{*} s^{\prime}$.

Example 3 (ACS Generated from Example 1). A (simplified) pictorial representation of the ACS generated by our procedure from the program in Example 1 (with the parametric entry point of Section 5) is shown in Figure 4, using a 0-CFA analysis. The three pid-classes correspond to the starting process $\widehat{\iota}_{0}$ and the two static calls of spawn in the program, the one for the shared cell process $\widehat{\iota}_{\mathrm{c}}$ and the other, $\widehat{\iota_{\mathrm{i}}}$, for all the processes running inc.

The first component of the ACS, the starting one, just spawns a shared cell and an arbitrary number of concurrent copies of the third component; these actions increment the counter associated with states 'res_free' and 'inc ${ }_{0}$ '. The second component represents the intended protocol quite closely; note that by abstracting messages they essentially become tokens and do not have a payload anymore. The rules of the third component clearly show its sequential behaviour. The entry point is $\left(\widehat{\iota}_{0}\right.$, cell_start $)$.

The VAS semantics is accurate enough in this case to prove mutual exclusion of, say, state ' $\mathrm{inc}_{2}$ ', which is protected by locks. Let's say for example that $n>0$ processes of pid-class $\widehat{\iota_{\mathrm{i}}}$ reached state 'inc ${ }_{1}$ '; each of them sent a lock message to the cell; note that now the message does not contain the pid of the requester so all these messages are indistinguishable; moreover the order of arrival is lost, we just count them. Suppose that $\widehat{\iota}_{\mathrm{c}}$ is in state 'res_free'; since the counter for lock is $n$ and hence not zero, the rule labeled with ?lock is enabled; however, once fired the counter for 'res_free' is zero and the rule is disabled. Now exactly one ack can be sent to the 'collective' mailbox of pid-class $\widehat{\iota_{\mathrm{i}}}$ so the rule receiving the ack is enabled; but as long as it is fired, the only ack message is consumed and no other $\widehat{\iota}_{\mathrm{i}}$ process can proceed. This holds until the lock is released and so on. Hence only one process at a time can be in state 'inc ${ }_{2}$ '. This property can be stated as a coverability problem: can inc $_{2}=2$ be covered? Since the VAS semantics is given in terms of a VAS, the property is decidable

\footnotetext{
${ }^{4}$ Labels are abbreviated to unclutter the picture; for example $\{$ init, , , $\}$ is abbreviated with init
} 

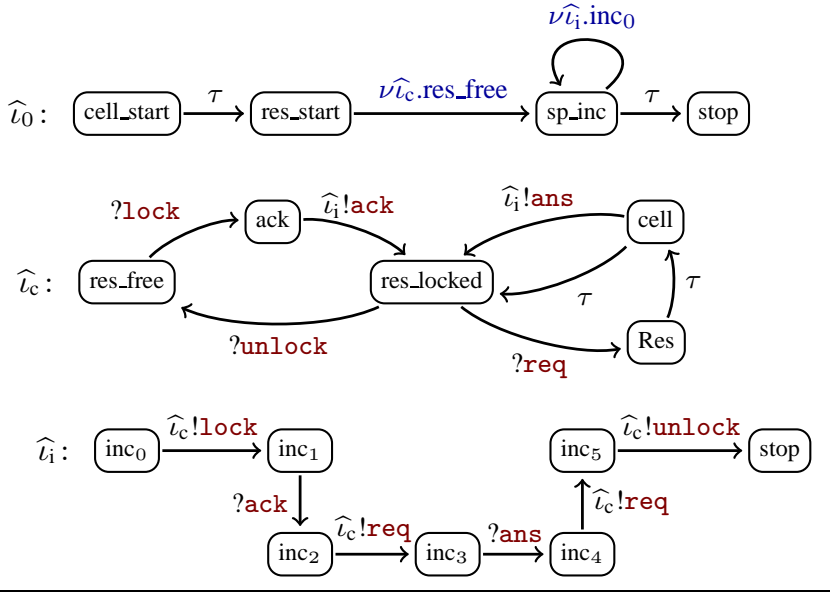

Figure 4. ACS generated by the algorithm from Example 1

and the answer can be algorithmically calculated. As we saw the answer is negative and then, by soundness, we can infer it holds in the actual semantics of the input program too.

Complexity of the Generation. Generating an ACS from a program amounts to calculating the analysis of Section 5 and aggregating the relevant ACS rules for each transition of the analysis. Since we are adding $O(1)$ rules to $R$ for each transition, the complexity of the generation is the same as the complexity of the analysis itself. The only reason for adding more than one rule to $R$ for a single transition is the cardinality of $\widehat{M s g}$ but since this costs only a constant overhead, increasing the precision with respect to message types is not as expensive as adopting more precise data abstractions.

Dimension of the Abstract Model. The complexity of coverability on VAS is EXPSPACE in the dimension of the VAS; hence for the approach to be practical, it is critical to keep the number of components of the VAS underlying the generated ACS small; in what follows we call dimension of an ACS the dimension of the VAS underlying its VAS semantics.

Our algorithm produces an ACS with dimension $(\mid$ ProcState $\mid+$ $|\widehat{M s g}|) \times|\widehat{P i d}|$. With the 0-CFA abstraction described at the end of Section 5, ProcState is polynomial in the size of the program and $\widehat{P i d}$ is linear in the size of the program so, assuming $|\widehat{M s g}|$ to be a constant, the dimension of the generated ACS is polynomial in the size of the program, in the worst case. Due to the parametricity of the abstract interpretation we can adjust for the right levels of precision and speed. For example, if the property at hand is not sensitive to pids, one can choose a coarser pid abstraction. It is also possible to greatly reduce ProcState: we observe that many of the control states result from intermediate functional reductions; such reduc-

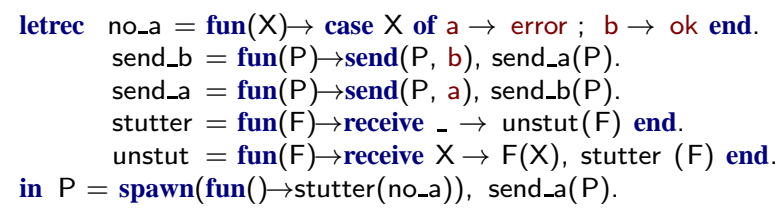

Figure 5. A program that Soter cannot verify because of the sequencing in mailboxes tions performed by different processes are independent, thanks to the actor model paradigm. This allows for the use of preorder reductions. In our prototype, as described in Section 7, we implemented a simple reduction that safely removes states which only represent internal functional transitions, irrelevant to the property at hand. This has proven to be a simple yet effective transformation yielding a significant speedup. We conjecture that, after the reduction, the cardinality of ProcState is quadratic only in the number of send, spawn and receive of the program.

\section{Evaluation, Limitations and Extensions}

Empirical Evaluation. To evaluate the feasibility of the approach, we have constructed Soter, a prototype implementation of our method for verifying Erlang programs. Written in Haskell, Soter takes as input a single Erlang module annotated with safety properties in the form of simple assertions. Soter supports the full higher-order fragment and the (single-node) concurrency and communication primitives of Erlang; features not supported by Soter are described in Remark 1. For more details about the tool see [11]. The annotated Erlang module is first compiled to Core Erlang by the Erlang compiler. A 0-CFA-like analysis, with support for the Data $_{D}$ data and message abstraction, is then performed on the compile; subsequently an ACS is generated. The ACS is simplified and then fed to the backend model-checker along with coverability queries translated from the annotations in the input Erlang program. Soter's backend is the tool BFC [18] which features a fast coverability engine for a variant of VAS. At the end of the verification pathway, if the answer is YES then the program is safe with respect to the input property, otherwise the analysis is inconclusive.

In Table 1 we summarise our experimental results. Many of the examples are higher-order and use dynamic (and unbounded) process creation and non-trivial synchronization. Example 1 appears as reslock and Soter proves mutual exclusion of the clients' critical section. concdb is the example program of [16] for which we prove mutual exclusion. pipe is inspired by the 'pipe' example of [19]; the property proved here is boundedness of mailboxes. sieve is a dynamically spawning higher-order concurrent implementation of Erathostene's sieve inspired by a program by Rob Pike; ${ }^{5}$ Soter can prove all the mailboxes are bounded.

All example programs, annotated with coverability queries, can be viewed and verified using Soter at http://mjolnir.cs.ox. ac.uk/soter/.

Limitations There are programs and properties that cannot be proved using any of the presented abstractions. (i) The program in Figure 5 defines a simple function that discards a message in the mailbox and feeds the next to its functional argument and so on in a loop. Another process sends a 'bad argument' and a good one in alternation such that only the good ones are fed to the function. The property is that the function is never called with a bad argument. This cannot be proved because sequential information of the mailboxes, which is essential for the verification, is lost in the counter abstraction. (ii) The program in Figure 6 defines a higher-order combinator that spawns a number of identical workers, each applied to a different task in a list. It then waits for all the workers to return a result before collecting them in a list which is subsequently returned. The desired property is that the combinator only returns when every worker has sent back its result. Unfortunately to prove this property, stack reasoning is required, which is beyond the capabilities of an ACS.

Refinement and Extensions. Our parametric definition of the abstract semantics allows us to tune the precision of the analysis

\footnotetext{
5 see "Concurrency and message passing in Newsqueak", http://youtu. be/hB05UFq0tFA
} 


\begin{tabular}{|c|c|c|c|c|c|c|c|c|c|c|c|}
\hline \multirow{2}{*}{ Example } & \multirow{2}{*}{ LOC } & \multirow{2}{*}{ PRP } & \multirow{2}{*}{ SAFE? } & \multicolumn{2}{|c|}{ ABSTR } & \multicolumn{2}{|c|}{ ACS SIZE } & \multicolumn{4}{|c|}{ TIME } \\
\hline & & & & $\mathrm{D}$ & $\mathrm{M}$ & Places & Ratio & Analysis & Simpl & $\mathrm{BFC}$ & Total \\
\hline reslock & 356 & 1 & yes & 0 & 2 & 40 & $10 \%$ & 0.56 & 0.08 & 0.82 & 1.48 \\
\hline sieve & 230 & 3 & yes & 0 & 2 & 47 & $19 \%$ & 0.26 & 0.03 & 2.46 & 2.76 \\
\hline concdb & 321 & 1 & yes & 0 & 2 & 67 & $12 \%$ & 1.10 & 0.16 & 5.19 & 6.46 \\
\hline state_factory & 295 & 2 & yes & 0 & 1 & 22 & $4 \%$ & 0.59 & 0.13 & 0.02 & 0.75 \\
\hline pipe & 173 & 1 & yes & 0 & 0 & 18 & $8 \%$ & 0.15 & 0.03 & 0.00 & 0.18 \\
\hline ring & 211 & 1 & yes & 0 & 2 & 36 & $9 \%$ & 0.55 & 0.07 & 0.25 & 0.88 \\
\hline parikh & 101 & 1 & yes & 0 & 2 & 42 & $41 \%$ & 0.05 & 0.01 & 0.07 & 0.13 \\
\hline unsafe_send & 49 & 1 & no & 0 & 1 & 10 & $38 \%$ & 0.02 & 0.00 & 0.00 & 0.02 \\
\hline safe_send & 82 & 1 & no* & 0 & 1 & 33 & $36 \%$ & 0.05 & 0.01 & 0.00 & 0.06 \\
\hline safe_send & 82 & 4 & yes & 1 & 2 & 82 & $34 \%$ & 0.23 & 0.03 & 0.06 & 0.32 \\
\hline firewall & 236 & 1 & no* & 0 & 2 & 35 & $10 \%$ & 0.36 & 0.05 & 0.02 & 0.44 \\
\hline firewall & 236 & 1 & yes & 1 & 3 & 74 & $10 \%$ & 2.38 & 0.30 & 0.00 & 2.69 \\
\hline finite_leader & 555 & 1 & no* & 0 & 2 & 56 & $20 \%$ & 0.35 & 0.03 & 0.01 & 0.40 \\
\hline finite_leader & 555 & 1 & yes & 1 & 3 & 97 & $23 \%$ & 0.75 & 0.07 & 0.86 & 1.70 \\
\hline stutter & 115 & 1 & no* & 0 & 0 & 15 & $19 \%$ & 0.04 & 0.00 & 0.00 & 0.05 \\
\hline howait & 187 & 1 & no* & 0 & 2 & 29 & $14 \%$ & 0.19 & 0.02 & 0.00 & 0.22 \\
\hline
\end{tabular}

Table 1. Soter Benchmarks. The number of lines of code refers to the compiled Core Erlang. The PRP column indicates the number of properties which need to be proved. The columns D and M indicate the data and message abstraction depth respectively. In the "Safe?" column, "no*" means that the program satisfies the properties but the verification was inconclusive; "no" means that the program is not safe and Soter finds a genuine counterexample. "Places" is the number of places of the underlying Petri net after the simplification; "Ratio" is the ratio of the number of places of the generated Petri net before and after the simplification. All times are in seconds.

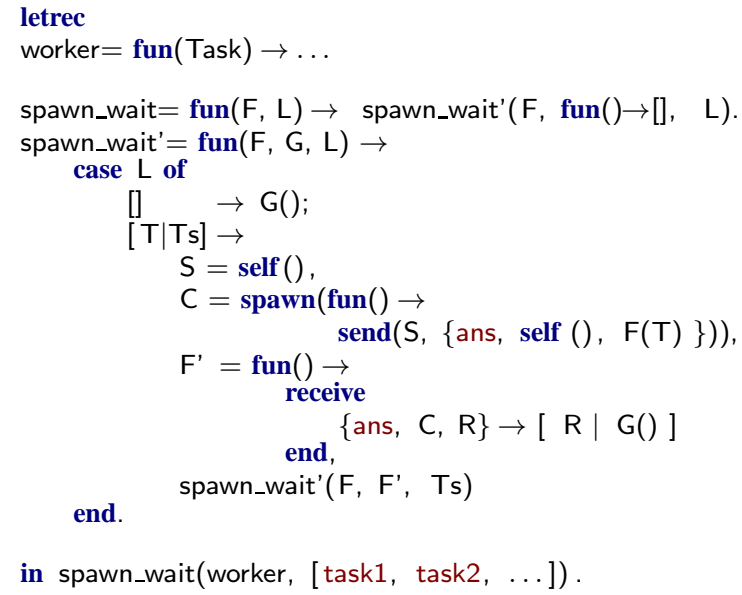

Figure 6. A program that Soter cannot verify because of the stack

when the abstraction is too coarse for the property to be proved. For safety properties, the counter-example witnessing a no-instance is a finite run of the abstract model. We conjecture that, given a spurious counter-example, it is possible to compute a suitable refinement of the basic domains abstraction so that the counter-example is no longer a run of the corresponding abstract semantics. However a naïve implementation of the refinement loop would suffer from state explosion. A feasible CEGAR loop will need to utilise sharper abstractions: it is possible for example to pinpoint a particular pid or call or mailbox for which the abstract domains need to be more precise while coarsely abstracting the rest. The development of a fully-fledged CEGAR loop is a topic of ongoing research.

The general architecture of our approach, combining static analysis and abstract model generation, can be adapted to accommodate different language features and different abstract models. By appropriate decoration of the analysis, it is possible to derive even more complex models for which semi-decision verification procedures have been developed [4, 21].

\section{Related Work}

Static Analysis. Verification or bug-finding tools for Erlang [6$8,20,22,27]$ typically rely on static analysis. The information obtained, usually in the form of a call graph, is then used to extract type constraints or infer runtime properties. Examples of static analyses of Erlang programs in the literature include data-flow [6], control-flow [20, 27] and escape [7] analyses.

Van Horn and Might [25] derive a CFA for a multithreaded extension of Scheme, using the same methodology [33] that we follow. The concurrency model therein is thread-based, and uses a compare-and-swap primitive. Our contribution, in addition to extending the methodology to Actor concurrency, is to use the derived parametric abstract interpretation to bootstrap the construction of an infinite-state abstract model for automated verification.

Reppy and Xiao [31] and Colby [9] analyse the channel communication patterns of Concurrent ML (CML). CML is based on typed channels and synchronous message passing, unlike the Actor-based concurrency model of Erlang.

Venet [34] proposed an abstract interpretation framework for the sanalysis of $\pi$-calculus, later extended to other process algebras by Feret [12] and applied to CAP, a process calculus based on the Actor model, by Garoche [15]. In particular, Feret's non-standard semantics can be seen as an alternative to Van Horn and Might's methodology, but tailored for process calculi.

Model Checking. Huch [16] uses abstract interpretation and model checking to verify LTL-definable properties of a restricted fragment of Erlang programs: (i) order-one (ii) tail-recursive (subsequently relaxed in a follow-up paper [17]), (iii) mailboxes are bounded (iv) programs spawn a fixed, statically computable, number of processes. Given a data abstraction function, his method transforms a program to an abstract, finite-state model; if a path property can be proved for the abstract model, then it holds for the input Erlang program. In contrast, our method can verify Erlang programs of every finite order, with no restriction on the size of mailboxes, or the number of processes that may be spawned. Since our method of verification is by transformation to a decidable infinite-state system that simulates the input program, it is capable of greater accuracy. 
McErlang is a model checker for Erlang programs developed by Fredlund and Svensson [14]. Given a program, a Büchi automaton, and an abstraction function, McErlang explores on-the-fly a product of an abstract model of the program and the Büchi automaton encoding a property. When the abstracted model is infinite-state, McErlang's exploration may not terminate. McErlang implements a fully-fledged Erlang runtime system, and it supports a substantial part of the language, including distributed and fault-tolerant features.

ACS can be expressed as processes in a suitable variant of CCS [26]. Decidable fragments of process calculi have been used in the literature to verify concurrent systems. Meyer [23] isolated a rich fragment of the $\pi$-calculus called depth-bounded. For certain patterns of communication, this fragment can be the basis of an abstract model that avoids the "merging" of mailboxes of the processes belonging to the same pid-class. Erlang programs however can express processes which are not depth bounded. We plan to address the automatic abstraction of arbitrary Erlang programs as depth-bounded process elsewhere.

Bug finding. Dialyzer $[7,8,20]$ is a popular bug finding tool, included in the standard Erlang / OTP distribution. Given an Erlang program, the tool uses flow and escape [29] analyses to detect specific error patterns. Building on top of Dialyzer's static analysis, success types are derived. Lindahl and Sagonas' success types [20] 'never disallow the use of a function that will not result in a type clash during runtime' and thus never generate false positives. Dialyzer puts to good use the type annotations that programmers do use in practice; it scales well and is effective in detecting 'discrepancies' in Erlang code. However, success typing cannot be used to verify program correctness.

Conclusion. We have defined a generic analysis for $\lambda$ ACTOR, and a way of extracting from the analysis a simulating infinite-state $a b-$ stract model in the form of an ACS, which can be automatically verified for coverability: if a state of the abstract model is not coverable then the corresponding concrete states of the input $\lambda$ AстоR program are not reachable. Our constructions are parametric on the abstractions for Time, Mailbox and Data, thus enabling different analyses (implementing varying degrees of precision with different complexity bounds) to be easily instantiated. In particular, with a 0-CFA-like specialisation of the framework, the analysis and generation of the ACS are computable in polynomial time. Further, the dimension of the resulting ACS is polynomial in the length of the input $\lambda$ ACTоR program, small enough for the verification problem to be tractable in many useful cases. The empirical results using our prototype implementation Soter are encouraging. They demonstrate that the abstraction framework can be used to prove interesting safety properties of non-trivial programs automatically. We believe that the proposed technique can easily be adapted to accommodate other languages and other abstract models. The level of generality at which the algorithm is defined seems to support the definition of a CEGAR loop readily, the formalisation of which is a topic for future work.

\section{References}

[1] G. Agha. Actors: a model of concurrent computation in distributed systems. MIT Press, Cambridge, MA, USA, 1986.

[2] J. Armstrong. Erlang. CACM, 53(9):68, 2010.

[3] J. Armstrong, R. Virding, and M. Williams. Concurrent programming in Erlang. Prentice Hall, 1993.

[4] A. Bouajjani, J. Esparza, and T. Touili. A generic approach to the static analysis of concurrent programs with procedures. In ACM SIGPLAN Notices, volume 38, pages 62-73, 2003.

[5] R. Carlsson. An introduction to Core Erlang. In Proceedings of the PLI'01 Erlang Workshop, 2001.
[6] R. Carlsson, K. Sagonas, and J. Wilhelmsson. Message analysis for concurrent programs using message passing. ACM TOPLAS, 2006.

[7] M. Christakis and K. Sagonas. Static detection of race conditions in erlang. PADL, pages 119-133, 2010.

[8] M. Christakis and K. Sagonas. Detection of asynchronous message passing errors using static analysis. PADL, pages 5-18, 2011.

[9] C. Colby. Analyzing the communication topology of concurrent programs. In PEPM, pages 202-213, 1995.

[10] E. D’Osualdo, J. Kochems, and C.-H. L. Ong. Verifying Erlang-style concurrency automatically. Technical report, University of Oxford DCS Technical Report, 2011. http://mjolnir.cs.ox.ac.uk/ soter/cpmrs. pdf.

[11] E. D'Osualdo, J. Kochems, and C.-H. L. Ong. Soter: an automatic safety verifier for Erlang. In Proceedings of the 2nd edition on Programming systems, languages and applications based on actors, agents, and decentralized control abstractions, AGERE! '12, pages 137-140. ACM, 2012.

[12] J. Feret. Abstract interpretation of mobile systems. Journal of Logic and Algebraic Programming, 63(1):59130, 2005.

[13] A. Finkel and P. Schnoebelen. Well-structured transition systems everywhere! Theoretical Computer Science, 256(1-2):63-92, 2001.

[14] L. Fredlund and H. Svensson. McErlang: a model checker for a distributed functional programming language. In ICFP, pages 125136, 2007.

[15] P. Garoche, M Pantel, and X. Thirioux. Static safety for an actor dedicated process calculus by abstract interpretation. In FMOODS, pages 78-92, 2006.

[16] F. Huch. Verification of Erlang programs using abstract interpretation and model checking. In ICFP, pages 261-272, 1999.

[17] F. Huch. Model checking Erlang programs - abstracting recursive function calls. 64:195-219, 2002.

[18] A. Kaiser, D. Kroening, and T. Wahl. Efficient coverability analysis by proof minimization. In CONCUR, 2012. www. cprover.org/bfc/.

[19] N. Kobayashi, M. Nakade, and A. Yonezawa. Static analysis of communication for asynchronous concurrent programming languages. Static Analysis, pages 225-242, 1995.

[20] T. Lindahl and K. Sagonas. Practical type inference based on success typings. In PPDP, pages 167-178, 2006.

[21] Z. Long, G. Calin, R. Majumdar, and R. Meyer. Language-Theoretic abstraction refinement. In FASE, pages 362-376, 2012.

[22] S. Marlow and P. Wadler. A practical subtyping system for Erlang. In ICFP, pages 136-149, 1997.

[23] R. Meyer. On boundedness in depth in the $\pi$-calculus. In Fifth Ifip International Conference On Theoretical Computer Science, pages 477-489, 2008.

[24] J. Midtgaard and T. Jensen. A calculational approach to control-flow analysis by abstract interpretation. Static Analysis, pages 347-362, 2008.

[25] M. Might and D. Van Horn. A family of abstract interpretations for static analysis of concurrent higher-order programs. Static Analysis, pages 180-197, 2011.

[26] R. Milner. A calculus of communicating systems, volume 92. SpringerVerlag Germany, 1980.

[27] S. Nyström. A soft-typing system for Erlang. In ACM Sigplan Erlang Workshop, pages 56-71, 2003.

[28] C.-H. L. Ong and S. J. Ramsay. Verifying higher-order functional programs with pattern-matching algebraic data types. In $P O P L$, pages 587-598, 2011.

[29] Y. G. Park and B. Goldberg. Escape analysis on lists. In ACM SIGPLAN Notices, volume 27, pages 116-127, 1992.

[30] C. Rackoff. The covering and boundedness problems for vector addition systems. Theoretical Computer Science, 6:223-231, 1978.

[31] J. H. Reppy and Y. Xiao. Specialization of CML message-passing primitives. In POPL, pages 315-326, 2007. 
[32] O. Shivers. Control-Flow Analysis of Higher-Order Languages. PhD thesis, Carnegie Mellon University, 1991.

[33] D. Van Horn and M. Might. Abstracting abstract machines. In ICFP, pages 51-62, 2010.

[34] Arnaud Venet. Abstract interpretation of the pi-calculus. In LOMAPS, pages $51-75,1996$ 


\section{A. Abstract Domains, Orders, Abstraction Functions and Abstract Auxiliary Functions}

Abstract Domains, Orders and Abstraction Functions:

$\widehat{\text { Pid }}:=$ ProgLoc $\times \widehat{\text { Time }}$

$\leq_{\text {pid }}:==\times \leq_{\mathrm{t}}$

$\alpha_{\text {pid }}:=\mathrm{id} \times \alpha_{\mathrm{t}}$

$\widehat{\operatorname{VAdd} r}:=\widehat{\text { Pid }} \times \operatorname{Var} \times \widehat{\text { Data }} \times \widehat{\text { Time }}$

$\leq_{\mathrm{va}}:=\leq_{\text {pid }} \times=\times \leq_{\mathrm{d}} \times \leq_{\mathrm{t}}$

$\alpha_{\mathrm{va}}:=\alpha_{\text {pid }} \times$ id $\times \alpha_{\mathrm{d}} \times \alpha_{\mathrm{t}}$

$\widehat{E n v}:=\operatorname{Var} \rightarrow \widehat{\operatorname{VAdd} r}$

$\widehat{\rho} \leq_{\text {env }} \widehat{\rho} \Longleftrightarrow \forall x \in \operatorname{Var} . \widehat{\rho}(x) \leq_{\text {va }} \widehat{\rho}^{\prime}(x)$

$\alpha_{\text {env }}(\rho)(x):=\alpha_{\text {va }}(\rho(x))$

$\widehat{K A d d r}:=\widehat{P i d} \times$ ProgLoc $\times \widehat{E n v} \times \widehat{\text { Time }}$

$\leq_{\mathrm{ka}}:=\leq_{\text {pid }} \times=\times \leq_{\text {env }} \times \leq_{\mathrm{t}}$

$\alpha_{\mathrm{ka}}:=\alpha_{\text {pid }} \times \mathrm{id} \times \alpha_{\mathrm{env}} \times \alpha_{\mathrm{t}}$

$\widehat{\text { Closure }}:=$ ProgLoc $\times \widehat{\text { Env }}$

$\leq_{\mathrm{cl}}:==\times \leq_{\mathrm{env}}$

$\alpha_{\mathrm{cl}}:=\mathrm{id} \times \alpha_{\mathrm{env}}$

$\widehat{\text { Value }}:=\widehat{\text { Closure }} \uplus \widehat{\text { Pid }}$

$\leq_{\mathrm{val}}:=\leq_{\mathrm{cl}}+\leq_{\mathrm{pid}}$

$\alpha_{\mathrm{val}}:=\alpha_{\mathrm{cl}}+\alpha_{\mathrm{pid}}$

ProcState $:=($ ProgLoc $\uplus \widehat{\text { Pid }}) \times \widehat{E n v} \times \widehat{\text { KAddr }} \times$ Time

$\leq_{\mathrm{ps}}:=\left(=+\leq_{\mathrm{pid}}\right) \times \leq_{\mathrm{env}} \times \leq_{\mathrm{ka}} \times \leq_{\mathrm{t}}$

$\alpha_{\mathrm{ps}}:=\left(\mathrm{id}+\alpha_{\mathrm{pid}}\right) \times \alpha_{\mathrm{env}} \times \alpha_{\mathrm{ka}} \times \alpha_{\mathrm{t}}$

$\widehat{\text { Procs }}:=\widehat{\text { Pid }} \rightarrow \mathscr{P}$ (ProcState $)$

$$
\widehat{\pi} \leq_{\text {proc }} \widehat{\pi}^{\prime} \Longleftrightarrow \forall \widehat{\iota} \in \widehat{P i d} \cdot \widehat{\pi}(\widehat{\iota}) \subseteq \widehat{\pi}^{\prime}(\widehat{\iota})
$$$$
\alpha_{\text {procs }}(\pi)(\widehat{\iota}):=\left\{\alpha_{\text {ps }}(\pi(\iota)) \mid \alpha_{\text {pid }}(\iota)=\widehat{\iota}\right\}
$$

Mailboxes $:=\widehat{\text { Pid }} \rightarrow \widehat{\text { Mailbox }}$

$$
\begin{aligned}
& \widehat{\mu} \leq_{\mathrm{ms}} \widehat{\mu}^{\prime} \Longleftrightarrow \forall \widehat{\iota} \in \widehat{P i d} \cdot \widehat{\mu}(\widehat{\iota}) \leq_{\mathrm{m}} \widehat{\mu}^{\prime}(\widehat{\iota}) \\
& \alpha_{\mathrm{ms}}(\mu)(\widehat{\iota}):=\bigsqcup\left\{\alpha_{\mathrm{m}}(\mu(\iota)) \mid \alpha_{\text {pid }}(\iota)=\widehat{\iota}\right\}
\end{aligned}
$$

$\widehat{\text { Store }}:=(\widehat{\text { VAddr }} \rightarrow \mathscr{P}(\widehat{\text { Value }})) \times(\widehat{\text { KAdd } r} \rightarrow \mathscr{P}(\widehat{\text { Kont }}))$

$\widehat{\sigma} \leq_{\mathrm{st}} \widehat{\sigma}^{\prime} \Longleftrightarrow \forall \widehat{b} \in \widehat{V A d d r} . \widehat{\sigma}(\widehat{b}) \subseteq \widehat{\sigma}^{\prime}(\widehat{b})$

$\forall \widehat{a} \in \widehat{K A d d r} . \widehat{\sigma}(\widehat{a}) \subseteq \widehat{\sigma}^{\prime}(\widehat{a})$

$\alpha_{\mathrm{st}}(\sigma)(\widehat{b}):=\left\{\alpha_{\mathrm{val}}(\sigma(b)) \mid \alpha_{\mathrm{va}}(b)=\widehat{b}\right\}, \widehat{b} \in \widehat{V A d d r}$

$\alpha_{\mathrm{st}}(\sigma)(\widehat{a}):=\left\{\alpha_{\mathrm{kont}}(\sigma(a)) \mid \alpha_{\mathrm{ka}}(a)=\widehat{a}\right\}, \widehat{a} \in \widehat{K A d d} r$

$\widehat{\text { State }}:=\widehat{\text { Procs }} \times$ Mailboxes $\times \widehat{\text { Store }}$

$$
\begin{aligned}
\leq & :=\leq_{\text {procs }} \times \leq_{\mathrm{ms}} \times \leq_{\mathrm{st}} \\
\alpha_{\mathrm{cfa}} & :=\left(\mathrm{id}+\alpha_{\mathrm{pid}}\right) \times \alpha_{\mathrm{env}}
\end{aligned}
$$

where we write $f+g:=\left\{\left(x, x^{\prime}\right) \mid\left(x, x^{\prime}\right) \in f\right.$ or $\left.\left(x, x^{\prime}\right) \in g\right\}$.

Abstract Auxiliary Functions:

$$
\begin{aligned}
& \text { newkpush }: \widehat{P i d} \times \text { ProcState } \rightarrow \widehat{\text { KAddr }} \\
& \widehat{n \mathrm{nWkpush}_{\mathrm{k}}}(\widehat{\iota},(\ell, \widehat{\rho},-, \widehat{t})):=\left(\widehat{\iota}, \ell \cdot \arg _{0}, \widehat{\rho}, \widehat{t}\right) \\
& \widehat{\text { new kpop }}: \widehat{\text { Pid }} \times \widehat{\text { Kont }} \times \text { ProcState } \rightarrow \widehat{\text { KAdd } r} \\
& \widehat{\text { newkpop }_{\text {k }}}\left(\widehat{\iota}, \widehat{\kappa},\left\langle_{-},{ }_{-},-, \widehat{t}\right\rangle\right):=\left(\widehat{\iota}, \ell \cdot \arg _{i+1}, \widehat{\rho}, \widehat{t}\right)
\end{aligned}
$$

where $\kappa=\operatorname{Arg}_{i}\left\langle\ell, \ldots, \widehat{\rho},{ }_{-}\right\rangle$

$\widehat{\text { new }_{\text {va }}}: \widehat{\text { Pid }} \times \operatorname{Var} \times \widehat{\text { Data }} \times$ ProcState $\rightarrow \widehat{\text { VAddr }}$

$\widehat{\text { new }_{\mathrm{va}}}\left(\widehat{\iota}, x, \widehat{\delta},\left\langle_{-},-,, \widehat{t}\right\rangle\right):=(\widehat{\iota}, x, \widehat{\delta}, \widehat{t})$

$\widehat{\text { new pid }}: \widehat{P i d} \times \operatorname{Prog} L o c \times \widehat{\text { Time }} \rightarrow \widehat{\text { Pid }}$

$\widehat{\text { newpid }_{\text {pid }}}\left(\left(\ell^{\prime}, \widehat{t}^{\prime}\right), \ell, \widehat{t}\right):=\left(\ell, \widehat{\operatorname{tick}}^{*}\left(\widehat{t}, \widehat{\operatorname{tick}}\left(\ell^{\prime}, \widehat{t}^{\prime}\right)\right)\right.$

Concrete and Abstract Match Function:

$$
\begin{aligned}
& \operatorname{match}_{\rho, \sigma}\left(p_{i},\left(x, \rho^{\prime}\right)\right)=\operatorname{match}_{\rho, \sigma}\left(p_{i}, \sigma\left(\rho^{\prime}(x)\right)\right) \\
& \operatorname{match}_{\rho, \sigma}(x, d)=\{x \mapsto d\} \text { if } x \notin \operatorname{dom}(\rho) \\
& \operatorname{match}_{\rho, \sigma}(x, d)=\{x \mapsto d\} \text { if } \operatorname{match}_{\rho^{\prime}, \sigma}\left(p^{\prime}, d\right) \neq \perp \\
& \text { where }\left(p^{\prime}, \rho^{\prime}\right)=\sigma(\rho(x)) \\
& \operatorname{match}_{\rho, \sigma}\left(p,\left(t, \rho^{\prime}\right)\right)=\bigotimes_{1 \leq i \leq n} \operatorname{match}_{\rho, \sigma}\left(p_{i},\left(t_{i}, \rho^{\prime}\right)\right) \\
& \text { where } \quad=c\left(p_{1}, \ldots, p_{n}\right) \\
& t=c\left(t_{1}, \ldots, t_{n}\right) \\
& \theta \otimes \theta^{\prime}=\perp \quad \text { if } \exists x . \theta(x) \neq \theta^{\prime}(x) \\
& \theta \otimes \theta^{\prime}=\theta \cup \theta^{\prime} \quad \text { otherwise } \\
& \bigotimes=[] \quad
\end{aligned}
$$

$\operatorname{match}_{\rho, \sigma}(p, d)=\perp \quad$ otherwise

$$
\begin{aligned}
& \widehat{\operatorname{match}}_{\widehat{\rho}, \widehat{\sigma}}\left(p_{i},\left(x, \hat{\rho}^{\prime}\right)\right)=\bigcup_{\widehat{d} \in \widehat{\sigma}\left(\widehat{\rho}^{\prime}(x)\right)} \widehat{\operatorname{match}}_{\widehat{\rho}, \widehat{\sigma}}\left(p_{i}, \widehat{d}\right) \\
& \widehat{\operatorname{match}}_{\widehat{\rho}, \widehat{\sigma}}(x, d)=\{x \mapsto \widehat{d}\} \\
& \widehat{\operatorname{match}}_{\widehat{\rho}, \widehat{\sigma}}\left(p,\left(t, \widehat{\rho}^{\prime}\right)\right)=\widehat{\bigotimes}_{1 \leq i \leq n} \widehat{\operatorname{match}}_{\widehat{\rho}, \widehat{\sigma}}\left(p_{i},\left(t_{i}, \widehat{\rho}^{\prime}\right)\right) \\
& \text { if } p=c\left(p_{1}, \ldots, p_{n}\right) \text { and } \\
& t=\mathrm{c}\left(t_{1}, \ldots, t_{n}\right)
\end{aligned}
$$

where $\widehat{\bigotimes}\left(\left\{\Theta_{i} \mid 1 \leq i \leq n\right\}\right)=\left\{\begin{array}{l}\theta \mid \begin{array}{l}\theta=\bigotimes_{1 \leq i \leq n} \theta_{i}, \theta \neq \perp, \\ \theta_{i} \in \Theta_{i}, 1 \leq i \leq n\end{array}\end{array}\right\}$

$\widehat{\operatorname{match}}_{\widehat{\rho}, \widehat{\sigma}}(p, d)=\emptyset \quad$ otherwise

Lemma 1. Suppose the concrete domain $C=A \rightarrow B$ of partial functions has abstract domain $\widehat{C}=\widehat{A} \rightarrow \mathscr{P}(\widehat{B})$ with the induced order $\leq$ and abstraction function $\alpha_{C}: C \rightarrow \widehat{C}$ as specified in 5 then for all $f \in C$ and for all $\alpha_{C}(f) \leq \widehat{f}$

$$
\forall a \in \operatorname{dom}(f) . \alpha_{B}(f(a)) \in \widehat{f}\left(\alpha_{A}(a)\right) .
$$

Further suppose $f, f^{\prime} \in C$ such that $f^{\prime}=f\left[a_{1} \mapsto b_{1}, \ldots, a_{n} \mapsto\right.$ $\left.b_{n}\right]$ and let $\widehat{f}, \widehat{f}^{\prime} \in \widehat{C}$ such that $\widehat{f}^{\prime}=\widehat{f} \sqcup\left[\widehat{a}_{1} \mapsto \widehat{b}_{1}, \ldots, \widehat{a}_{n} \mapsto \widehat{b}_{n}\right]$ with $\alpha_{C}(f) \leq \widehat{f}$ and $\alpha_{A}\left(a_{i}\right)=\widehat{a}_{i}, \alpha_{B}\left(b_{i}\right)=\widehat{b}_{i}$ for $i=1, \ldots, n$ then

$$
\alpha_{C}\left(f^{\prime}\right) \leq \widehat{f}^{\prime} .
$$

Proof. Let $f \in C$ and $\widehat{f} \in \widehat{C}$ such that $\alpha_{\mathrm{C}}(f) \leq \widehat{f}$. The definition of $\leq$ implies that for all $\widehat{a} \in \widehat{A}$

$$
\alpha_{\mathrm{C}}(f)(\widehat{a}) \subseteq \widehat{f}(\widehat{a}) .
$$

Take $a \in A$ and fix $\widehat{a}=\alpha_{\mathrm{A}}(a)$ then we obtain

$$
\alpha_{\mathrm{C}}(f)\left(\alpha_{\mathrm{A}}(a)\right) \subseteq \widehat{f}\left(\alpha_{\mathrm{A}}(a)\right) .
$$


Expanding the definition of $\alpha_{\mathrm{C}}$ yields

$$
\left.\left\{\alpha_{\mathrm{B}}\left(b_{0}\right) \mid\left(a_{0}, b_{0}\right) \in f, \alpha_{\mathrm{A}}\left(a_{0}\right)=\alpha_{\mathrm{A}}(a)\right)\right\} \subseteq \widehat{f}\left(\alpha_{\mathrm{A}}(a)\right) .
$$

In particular $\alpha_{\mathrm{B}}(f(a)) \in\left\{\alpha_{\mathrm{B}}\left(b_{0}\right) \mid\left(a_{0}, b_{0}\right) \in f, \alpha_{\mathrm{A}}\left(a_{0}\right)=\right.$ $\left.\left.\alpha_{\mathrm{A}}(a)\right)\right\}$ which yields what we set out to prove

$$
\forall a \in \operatorname{dom}(f) . \alpha_{\mathrm{B}}(f(a)) \in \widehat{f}\left(\alpha_{\mathrm{A}}(a)\right) .
$$

Turning to equation 7 we want to show $\alpha_{\mathrm{C}}\left(f^{\prime}\right) \leq \widehat{f}^{\prime}$. Let $\widehat{a} \in \widehat{A}$ then there are several cases to consider

(i) $\alpha_{\mathrm{C}}\left(f^{\prime}\right)(\widehat{a})=\alpha_{\mathrm{C}}(f)(\widehat{a})$. Then since $\alpha_{\mathrm{C}}(f) \leq \widehat{f} \leq \widehat{f}^{\prime}$ we have $\alpha_{\mathrm{C}}(f)(\widehat{a}) \subseteq \widehat{f}(\widehat{a}) \subseteq \widehat{f}^{\prime}(\widehat{a})$.

(ii) $\widehat{a}=\alpha_{\mathrm{A}}\left(a_{i}\right)$ for some $1 \leq i \leq n$. Then $\widehat{a}=\widehat{a}_{i}$ and thus

$$
\begin{aligned}
\alpha_{\mathrm{C}}\left(f^{\prime}\right)(\widehat{a}) & =\left\{\alpha_{\mathrm{B}}\left(b_{i}\right)\right\} \cup\left\{\alpha_{\mathrm{B}}(f(a)) \mid \alpha_{\mathrm{A}}(a)=\widehat{a}, a \neq a_{i}\right\} \\
& \subseteq\left\{\widehat{b}_{i}\right\} \cup \alpha_{\mathrm{C}}(f)(\widehat{a}) \\
& \subseteq\left\{\widehat{b}_{i}\right\} \cup \widehat{f}(\widehat{a}) \subseteq \widehat{f}^{\prime}(\widehat{a})
\end{aligned}
$$

(iii) otherwise there does not exist $(a, b) \in f^{\prime}$ such that $\alpha_{\mathrm{A}}(a)=\widehat{a}$ and hence $\alpha_{\mathrm{C}}\left(f^{\prime}\right)(a)=\emptyset$ which makes our claim trivially true.

We can thus conclude that $\alpha_{\mathrm{C}}\left(f^{\prime}\right) \leq \widehat{f}^{\prime}$.

Corollary 3. Let $\pi \in$ Procs and $\widehat{\pi} \in \widehat{\text { Procs }}$ such that $\alpha_{\text {proc }}(\pi) \leq$ $\widehat{\pi}$, let $\sigma \in$ Store and $\widehat{\sigma} \in \widehat{\text { Store }}$ such that $\alpha_{s t}(\sigma) \leq \widehat{\sigma}$ and Let $\mu \in$ Mailboxes and $\widehat{\mu} \in$ Mailboxes such that $\alpha_{m s}(\mu) \leq \widehat{\mu}$ then

(i) $\forall \iota \in P i d . \alpha_{p s}(\pi(\iota)) \in \widehat{\pi}\left(\alpha_{\text {pid }}(\iota)\right)$

(ii) $\forall b \in V A d d r . \alpha_{\text {val }}(\sigma(b)) \in \widehat{\sigma}\left(\alpha_{v a}(b)\right)$

(iii) $\forall a \in K A d d r . \alpha_{\text {kont }}(\sigma(a)) \in \widehat{\sigma}\left(\alpha_{k a}(a)\right)$

(iv) $\forall \iota \in P i d . \alpha_{m}(\mu(\iota)) \leq \widehat{\mu}\left(\alpha_{\text {pid }}(\iota)\right)$

(v) $\forall \iota \in$ Pid. $\forall x \in$ Var.$\forall \delta \in$ Data. $\forall q \in$ ProcState .

$$
\alpha_{v a}\left(\operatorname{new}_{v a}(\iota, x, \delta, q)\right)=\widehat{\operatorname{new}_{v a}}\left(\alpha_{p i d}(\iota), x, \alpha_{d}(\delta), \alpha_{p s}(q)\right)
$$

Proof. Cases (i) - (iii) follow directly from Lemma 1; it remains to show the claims of (iv) and (v).

(iv) By assumption $\alpha_{\mathrm{ms}}(\mu) \leq \widehat{\mu}$ which implies that

$$
\alpha_{\mathrm{ms}}(\mu)\left(\alpha_{\text {pid }}(\iota)\right) \leq \widehat{\mu}\left(\alpha_{\text {pid }}(\iota)\right)=\widehat{\mu}(\widehat{\iota}) .
$$

Expanding $\alpha_{\mathrm{ms}}$ then gives us that $\alpha_{\mathrm{m}}(\mu(\iota)) \leq \alpha_{\mathrm{ms}}(\mu)\left(\alpha_{\text {pid }}(\iota)\right)$, since $\alpha_{\mathrm{ms}}(\mu)=\lambda \widehat{\iota} . \bigsqcup\left\{\alpha_{\mathrm{m}}(\mu(\iota)) \mid \alpha_{\text {pid }}(\iota)=\widehat{\iota}\right\}$, which allows us to conclude

$$
\alpha_{\mathrm{m}}(\mu(\iota)) \leq \widehat{\mu}(\widehat{\iota}) .
$$

(v) The claim follows straightforwardly from expanding new $\mathrm{w}_{\mathrm{va}}$ and $\widehat{\text { newva }}$ :

$$
\begin{aligned}
\alpha_{\mathrm{va}}\left(\operatorname{new}_{\mathrm{va}}(\iota, x, \delta, q)\right) & =\left(\alpha_{\text {pid }}(\iota), x, \alpha_{\mathrm{d}}(\delta), \alpha_{\mathrm{t}}(t)\right) \\
& \widehat{\operatorname{new}_{\mathrm{va}}}\left(\alpha_{\text {pid }}(\iota), x, \alpha_{\mathrm{d}}(\delta), \alpha_{\mathrm{ps}}(q)\right)
\end{aligned}
$$

where $q=\langle e, \rho, a, t\rangle$.

\section{B. Proof of Theorem 1}

Proof of Theorem 1. The proof is by a case analysis of the rule that defines the concrete transition $s \rightarrow s^{\prime}$. For each rule, the transition in the concrete system can be replicated in the abstract transition system using the abstract version of the rule, with the appropriate choice of abstract pid, the continuation from the abstract store, the message from the abstract mailbox, etc.

Let $s=\langle\pi, \mu, \sigma\rangle \rightarrow\left\langle\pi^{\prime}, \mu^{\prime}, \sigma^{\prime}\right\rangle=s^{\prime}$ and $u=\langle\widehat{\pi}, \widehat{\mu}, \widehat{\sigma}\rangle$ such that $\alpha_{\text {proc }}(\pi) \leq \widehat{\pi}, \alpha_{\text {mail }}(\mu) \leq \widehat{\mu}$, and $\alpha_{\text {st }}(\sigma) \leq \widehat{\sigma}$. We consider a number of rules for illustration.
Case: (Send). We know that $s \rightarrow s^{\prime}$ using rule Send; we can thus assume

$$
\begin{aligned}
\pi(\iota) & =\langle v, \rho, a, t\rangle \\
\sigma(a) & =\operatorname{Arg}_{2}\left\langle\ell, d, \iota^{\prime},{ }_{-}, c\right\rangle \\
d & =(\text { send, },-)
\end{aligned}
$$

and for $s^{\prime}$

$$
\begin{aligned}
\pi^{\prime} & =\pi[\iota \mapsto\langle v, \rho, c, t\rangle] \\
\mu^{\prime} & =\mu\left[\iota^{\prime} \mapsto \operatorname{enq}\left((v, \rho), \mu\left(\iota^{\prime}\right)\right)\right] \\
\sigma^{\prime} & =\sigma .
\end{aligned}
$$

As a first step we will examine $u$ and show that $u \rightsquigarrow u^{\prime}$ for some $u^{\prime}$. For $\widehat{\pi}$ and $\widehat{\sigma}$, writing $\widehat{\iota}:=\alpha_{\text {pid }}(\iota)$, Corollary 3 gives us

$$
\begin{aligned}
& \langle v, \widehat{\rho}, \widehat{a}, \widehat{t}\rangle \in \widehat{\pi}(\widehat{\iota}) \\
& \operatorname{Arg}_{2}\langle\ell, \widehat{d}, \widehat{\iota},,, \widehat{c}\rangle \in \widehat{\sigma}(\widehat{a})
\end{aligned}
$$

where $\alpha_{\text {env }}(\rho)=\widehat{\rho}, \alpha_{\text {ak }}(a)=\widehat{a}, \widehat{d}=\alpha_{\text {val }}(d), \widehat{t}=\alpha_{\mathrm{t}}(t)$, $\widehat{\iota}=\alpha_{\text {pid }}\left(\iota^{\prime}\right)$ and $\widehat{c}=\alpha_{\mathrm{ka}}(c)$. Rule AbsSend is now applicable and we can set

$$
\begin{aligned}
& \widehat{\pi}^{\prime}:=\widehat{\pi} \sqcup[\widehat{\iota} \mapsto \widehat{q}] \\
& \widehat{q}:=\langle v, \widehat{\rho}, \widehat{c}, \widehat{t}\rangle \\
& \widehat{\mu}^{\prime}:=\widehat{\mu}\left[\widehat{\iota} \mapsto \widehat{\operatorname{enq}}\left((v, \widehat{\rho}), \widehat{\mu}\left(\widehat{\iota}^{\prime}\right)\right)\right] \\
& u^{\prime}:=\left\langle\widehat{\pi}^{\prime}, \widehat{\mu}^{\prime}, \widehat{\sigma}\right\rangle .
\end{aligned}
$$

It follows from rule (AbsSend) that $u \rightsquigarrow u^{\prime}$. It remains to show that $\alpha_{\mathrm{cfa}}\left(s^{\prime}\right) \leq u^{\prime}$ which follows directly from (i) $\alpha_{\text {proc }}\left(\pi^{\prime}\right) \leq \widehat{\pi}^{\prime}$ and (ii) $\alpha_{\mathrm{ms}}\left(\mu^{\prime}\right) \leq \widehat{\mu}^{\prime}$.

(i) $\alpha_{\text {proc }}\left(\pi^{\prime}\right) \leq \widehat{\pi}^{\prime}$ follows immediately from Lemma 1 since $\widehat{\iota}=\alpha_{\text {pid }}(\iota)$ and $\alpha_{\mathrm{ps}}(q)=\widehat{q}$.

(ii) $\alpha_{\mathrm{ms}}\left(\mu^{\prime}\right) \leq \widehat{\mu}^{\prime}$. It is sufficient to show that $\alpha_{\text {pid }}\left(\iota^{\prime}\right)=\widehat{\iota}$, which is immediate, and $\alpha_{\mathrm{m}}\left(\mu^{\prime}\left(\iota^{\prime}\right)\right) \leq \widehat{\mu}(\widehat{\iota})$. For the latter, since $\alpha_{\text {env }}(\rho)=\widehat{\rho}$, a sound basic domain abstraction gives us

$$
\begin{aligned}
\alpha_{\mathrm{m}}\left(\mu^{\prime}\left(\iota^{\prime}\right)\right) & =\alpha_{\mathrm{m}}\left(\operatorname{enq}\left((v, \rho), \mu\left(\iota^{\prime}\right)\right)\right) \\
& \leq \widehat{\operatorname{enq}}((v, \widehat{\rho}), \widehat{\mu}(\widehat{\iota}))=\widehat{\mu}(\widehat{\iota})
\end{aligned}
$$

provided we can show $\alpha_{\mathrm{m}}\left(\mu\left(\iota^{\prime}\right)\right) \leq \widehat{\mu}\left(\widehat{\iota}^{\prime}\right)$; the latter inequality follows Corollary 3 . Hence we can conclude $\alpha_{\mathrm{ms}}\left(\mu^{\prime}\right) \leq \widehat{\mu}^{\prime}$ which completes the proof of this case.

Case: (Receive). In the concrete $s \rightarrow s^{\prime}$ using the Receive, hence we can make the following assumptions

$$
\begin{aligned}
\pi(\iota) & =\left\langle\text { receive } p_{1} \rightarrow e_{1} \ldots p_{n} \rightarrow e_{n} \text { end }, \rho, a, t\right\rangle=: q \\
(i, \theta, \mathfrak{m}) & =\operatorname{mmatch}\left(p_{1} \ldots p_{n}, \mu(\iota), \rho, \sigma\right) \\
\theta & =\left[x_{1} \mapsto d_{1} \ldots x_{k} \mapsto d_{k}\right] \\
b_{j} & =\operatorname{new}_{\mathrm{va}}\left(\iota, x_{j}, \delta_{j}, q\right) \\
\delta_{j} & =\operatorname{res}\left(\sigma, d_{j}\right)
\end{aligned}
$$

and for state $s^{\prime}$

$$
\begin{aligned}
\pi^{\prime} & =\pi\left[\iota \mapsto q^{\prime}\right] \\
q^{\prime} & =\left\langle e_{i}, \rho^{\prime}, a, t\right\rangle \\
\rho^{\prime} & =\rho\left[x_{1} \mapsto b_{1} \ldots x_{k} \mapsto b_{k}\right] \\
\mu^{\prime} & =\mu[\iota \mapsto \mathfrak{m}] \\
\sigma^{\prime} & =\sigma\left[b_{1} \mapsto d_{1} \ldots b_{k} \mapsto d_{k}\right] .
\end{aligned}
$$

As a first step we will look at $u$ to prove there there exists a $u^{\prime}$ such that $u \rightsquigarrow u^{\prime}$ using rule AbsReceive. We can invoke Corollary 
3 , since $\alpha_{\text {proc }}(\pi) \leq \widehat{\pi}$, to obtain

$$
\widehat{q}:=\left\langle\text { receive } p_{1} \rightarrow e_{1} \ldots p_{n} \rightarrow e_{n} \text { end, } \widehat{\rho}, \widehat{a}, \widehat{t}\right\rangle \in \widehat{\pi}(\widehat{\iota})
$$

where we write $\widehat{\rho}:=\alpha_{\text {env }}(\rho), \widehat{a}:=\alpha_{\mathrm{ka}}(a), \widehat{t}=\alpha_{\mathrm{t}}(t)$ and $\widehat{\iota}:=\alpha_{\text {pid }}(\iota)$. Moreover Corollary 3 gives us

$$
\alpha_{\mathrm{m}}(\mu(\iota)) \leq \widehat{\mu}(\widehat{\iota}) .
$$

as $\alpha_{\mathrm{ms}}(\mu) \leq \widehat{\mu}$ and $\widehat{\iota}=\alpha_{\text {pid }}(\iota)$. Since the instantiation of the basic domains is sound and $\alpha_{\mathrm{st}}(\sigma) \leq \widehat{\sigma}$ we then know that

$$
(i, \widehat{\theta}, \widehat{\mathfrak{m}}) \in \widehat{\operatorname{mmatch}}(\vec{p}, \widehat{\mu}(\widehat{\iota}), \widehat{\rho}, \widehat{\sigma})
$$

such that $\widehat{\theta}=\alpha_{\text {sub }}(\theta)$ and $\widehat{\mathfrak{m}} \geq \alpha_{\mathrm{m}}(\mathfrak{m})$. Turning to the substitution $\widehat{\theta}$ we can see that

$$
\widehat{\theta}=\left[x_{1} \mapsto \widehat{d}_{1} \ldots x_{k} \mapsto \widehat{d}_{k}\right]
$$

where $\widehat{d}_{i}=\alpha\left(d_{i}\right)$ for $1 \leq i \leq k$. Appealing to the sound basic domain instantiation once more, noting that $\alpha_{\text {st }}(\sigma) \leq \widehat{\sigma}$, yields that for $j=1, \ldots, k$ we have $\widehat{\delta}_{j}:=\alpha_{\mathrm{d}}\left(\delta_{j}\right) \in \operatorname{res}\left(\widehat{\sigma}, \widehat{d}_{j}\right)$; to obtain new abstract variable addresses we can now set $\widehat{b}_{j}:=$ $\widehat{\text { new }_{\text {va }}}\left(\widehat{\iota}, x_{j}, \widehat{\delta}_{j}, \widehat{q}\right)$. Rule AbsReceive is applicable now; we make the following definitions

$$
\begin{aligned}
\widehat{\pi}^{\prime} & :=\widehat{\pi} \sqcup\left[\widehat{\iota} \mapsto \widehat{q}^{\prime}\right] \\
\widehat{q}^{\prime} & :=\left\langle e_{i}, \widehat{\rho}, \widehat{a}, \widehat{t}\right\rangle \\
\widehat{\rho}^{\prime} & :=\widehat{\rho}\left[x_{1} \mapsto \widehat{b}_{1} \ldots x_{k} \mapsto \widehat{b}_{k}\right] \\
\widehat{\mu}^{\prime} & :=\widehat{\mu}[\widehat{\imath} \mapsto \widehat{\mathfrak{m}}] \\
\widehat{\sigma}^{\prime} & :=\widehat{\sigma} \sqcup\left[\widehat{b}_{1} \mapsto \widehat{d}_{1} \ldots \widehat{b}_{k} \mapsto \widehat{d}_{k}\right] \\
\widehat{u}^{\prime} & :=\left\langle\widehat{\pi}^{\prime}, \widehat{\mu}^{\prime}, \widehat{\sigma}^{\prime}, \widehat{\vartheta}\right\rangle
\end{aligned}
$$

and observe that $u \rightsquigarrow u^{\prime}$. It remains to show $\alpha_{\mathrm{cfa}}\left(s^{\prime}\right) \leq u^{\prime}$ which follows directly if we can prove (i) $\alpha_{\text {proc }}\left(\pi^{\prime}\right) \leq \widehat{\pi}^{\prime}$, (ii) $\alpha_{\mathrm{ms}}\left(\mu^{\prime}\right) \leq \widehat{\mu}^{\prime}$ and (iii) $\alpha_{\mathrm{st}}\left(\sigma^{\prime}\right) \leq \widehat{\sigma}^{\prime}$.

(i) $\alpha_{\text {proc }}\left(\pi^{\prime}\right) \leq \widehat{\pi}^{\prime}$. We note that by Corollary 3 we know $\widehat{b}_{i}=$ $\alpha_{\mathrm{va}}\left(b_{i}\right)$ as $\widehat{\iota}=\alpha_{\text {pid }}(\iota), \widehat{\delta}_{i}=\alpha_{\mathrm{d}}\left(\delta_{i}\right)$ and $\alpha_{\text {proc }}(q)=\widehat{q}$ for $1 \leq i \leq n$. It follows that $\widehat{\rho}^{\prime}=\alpha_{\text {env }}\left(\rho^{\prime}\right)$ and hence $\widehat{q}^{\prime}=$ $\alpha_{\mathrm{ps}}\left(q^{\prime}\right)$. Lemma 1 is now applicable, since $\widehat{\iota}=\alpha_{\text {pid }}(\iota)$, to give $\alpha_{\text {proc }}\left(\pi^{\prime}\right) \leq \widehat{\pi}^{\prime}$.

(ii) $\alpha_{\mathrm{ms}}\left(\mu^{\prime}\right) \leq \widehat{\mu}^{\prime}$. It is sufficient to show that $\widehat{\iota}=\alpha_{\text {pid }}(\iota)$, which is immediate, and $\alpha_{\mathrm{m}}(\mathfrak{m}) \leq \widehat{\mathfrak{m}}$ which we have already established above; hence we can conclude $\alpha_{\mathrm{ms}}\left(\mu^{\prime}\right) \leq \widehat{\mu}^{\prime}$.

(iii) $\alpha_{\mathrm{st}}\left(\sigma^{\prime}\right) \leq \widehat{\sigma}^{\prime}$. The observation that $\widehat{b}_{i}=\alpha_{\mathrm{va}}\left(b_{i}\right)$ and $\widehat{d}_{i}=$ $\alpha_{\text {val }}\left(d_{i}\right)$ allows the application of Lemma 1 which gives $\alpha_{\text {st }}\left(\sigma^{\prime}\right) \leq$ $\widehat{\sigma}^{\prime}$ as desired.

This completes the proof of this case.

Case: (Apply). Since $s \rightarrow s^{\prime}$ using rule Apply we can assume that

$$
\begin{aligned}
\pi(\iota) & =\langle v, \rho, a, t\rangle=: q \\
\sigma(a) & =\operatorname{Arg}_{n}\left\langle\ell, d_{0} \ldots d_{n-1}, \rho^{\prime}, c\right\rangle:=\kappa \\
\operatorname{arity}(\ell) & =n \\
d_{0} & =\left(\mathbf{f u n}\left(x_{1} \ldots x_{n}\right) \rightarrow e, \rho_{0}\right) \\
d_{n} & =(v, \rho)
\end{aligned}
$$

and for $i=1, \ldots, n$

$$
\begin{aligned}
\delta_{i} & =\operatorname{res}\left(\sigma, d_{i}\right) \\
b_{i} & =\operatorname{new}_{\mathrm{va}}\left(\iota, x_{i}, \delta_{i}, q\right)
\end{aligned}
$$

additionally for the successor state $s^{\prime}$

$$
\pi^{\prime}=\pi\left[\iota \mapsto q^{\prime}\right]
$$

$$
\begin{aligned}
& \quad \text { where } q^{\prime}:=\left\langle e, \rho^{\prime}\left[x_{1} \rightarrow b_{1} \ldots x_{n} \rightarrow b_{n}\right], c, \operatorname{tick}(\ell, t)\right\rangle \\
& \sigma^{\prime}=\sigma\left[b_{1} \mapsto d_{1} \ldots b_{n} \mapsto d_{n}\right] \\
& \mu^{\prime}=\mu
\end{aligned}
$$

As a first step we will examine $u$ and show that there exists a $u^{\prime}$ such that $u \rightsquigarrow u^{\prime}$ using rule AbsApply. From Corollary 3, since $\alpha_{\text {proc }}(\pi) \leq \widehat{\pi}$, it follows that

$$
\widehat{q}:=\left\langle v, \alpha_{\text {env }}(\rho), \alpha_{\text {ka }}(a), \alpha_{\mathrm{t}}(t)\right\rangle \in \widehat{\pi}\left(\alpha_{\text {pid }}(\iota)\right) .
$$

Letting $\widehat{\rho}:=\alpha_{\text {env }}(\rho), \widehat{a}:=\alpha_{\text {ka }}(a), \widehat{t}:=\alpha_{\mathrm{t}}(t)$ and $\widehat{\iota}:=\alpha_{\text {pid }}(\iota)$ we can appeal to Corollary 3 again, as $\alpha_{\text {st }}(\sigma) \leq \widehat{\sigma}$, to obtain

$$
\operatorname{Arg}_{n}\left\langle\ell, \widehat{d}_{0} \ldots \widehat{d}_{n-1}, \widehat{\rho}^{\prime}, \widehat{c}\right\rangle \in \widehat{\sigma}(\widehat{a})
$$

where we write $\widehat{d}_{i}:=\alpha_{\text {val }}\left(d_{i}\right)$ for $0 \leq i<n, \widehat{\rho}^{\prime}:=\alpha_{\text {env }}\left(\rho^{\prime}\right)$ and $\widehat{c}:=\alpha_{\mathrm{ka}}(c)$. Expanding $\alpha_{\mathrm{val}}$ yields

$$
\widehat{d}_{0}=\left(\operatorname{fun}\left(x_{1} \ldots x_{n}\right) \rightarrow e, \widehat{\rho}_{0}\right)
$$

where we write $\widehat{\rho}_{0}:=\alpha_{\text {env }}\left(\rho_{0}\right)$. Taking $\widehat{d}_{n}:=(v, \widehat{\rho})$ we obtain from our sound basic domain abstraction

$$
\widehat{\delta}_{i}:=\alpha_{\mathrm{d}}\left(\delta_{i}\right) \in \widehat{\operatorname{res}}\left(\widehat{\sigma}, \widehat{d}_{i}\right) \text { for } i=1, \ldots, n
$$

as $\alpha_{\mathrm{st}}(\sigma) \leq \widehat{\sigma}$ and $\widehat{d}_{i}=\alpha_{\mathrm{val}}\left(d_{i}\right)$. Turning to the abstract variable addresses we define

$$
\widehat{b}_{i}:=\widehat{\operatorname{new}_{\mathrm{va}}}\left(\widehat{\iota}, x_{i}, \widehat{\delta}_{i}, \widehat{q}\right) \text { for } 1 \leq i \leq n .
$$

Rule AbsApply is now applicable and we define

$$
\begin{aligned}
\widehat{\pi}^{\prime} & :=\widehat{\pi} \sqcup\left[\widehat{\imath} \mapsto \widehat{q}^{\prime}\right] \\
\widehat{q}^{\prime} & :=\left\langle e, \widehat{\rho}^{\prime}\left[x_{1} \rightarrow \widehat{b}_{1} \ldots x_{n} \rightarrow \widehat{b}_{n}\right], \widehat{c}, \widehat{\operatorname{tick}}(\ell, \widehat{t})\right\rangle \\
\widehat{\sigma}^{\prime} & :=\widehat{\sigma} \sqcup\left[\widehat{b}_{1} \mapsto \widehat{d}_{1} \ldots \widehat{b}_{n} \mapsto \widehat{d}_{n}\right] \\
u^{\prime} & :=\left\langle\widehat{\pi}^{\prime}, \widehat{\mu}, \widehat{\sigma}^{\prime}\right\rangle .
\end{aligned}
$$

It is clear from rule AbsApply that $u \rightsquigarrow u^{\prime}$; it remains to show that $\alpha_{\mathrm{cfa}}\left(s^{\prime}\right) \leq u^{\prime}$ to prove this case. The latter follows if we can justify (i) $\alpha_{\text {proc }}\left(\bar{\pi}^{\prime}\right) \leq \widehat{\pi}^{\prime}$ and (ii) $\alpha_{\text {st }}\left(\sigma^{\prime}\right) \leq \widehat{\sigma}^{\prime}$.

(i) $\alpha_{\text {proc }}\left(\pi^{\prime}\right) \leq \widehat{\pi}^{\prime}$. We can appeal to Lemma 1 provided we can show that $\widehat{\iota}=\alpha_{\text {pid }}(\iota)$ and $\alpha_{\mathrm{ps}}\left(q^{\prime}\right)=\widehat{q}^{\prime}$ where the former is immediate. For the latter, first observe that since we have a sound basic domain abstraction we know $\alpha_{\mathrm{t}}(\operatorname{tick}(\ell, t)) \leq$ $\widehat{\operatorname{tick}}(\ell, \widehat{t})$; however as Time is a flat domain so the above inequality is in fact an equality

$$
\alpha_{\mathrm{t}}(\operatorname{tick}(\ell, t))=\widehat{\operatorname{tick}}(\ell, \widehat{t}) .
$$

Moreover $\widehat{b}_{i}=\alpha_{\mathrm{va}}\left(b_{i}\right)$ for $1 \leq i \leq n$ by Corollary 3 , hence

$$
\alpha_{\text {env }}\left(\rho^{\prime}\left[x_{1} \rightarrow b_{1} \ldots x_{n} \rightarrow b_{n}\right]\right)=\widehat{\rho}^{\prime}\left[x_{1} \rightarrow \widehat{b}_{1} \ldots x_{n} \rightarrow \widehat{b}_{n}\right]
$$

as $\alpha_{\text {env }}\left(\rho^{\prime}\right)=\widehat{\rho}^{\prime}$; in combination with $\widehat{c}=\alpha_{\text {ka }}(c)$ we obtain the desired $\alpha_{\mathrm{ps}}\left(q^{\prime}\right)=\widehat{q}^{\prime}$. Thus we conclude that $\alpha_{\text {proc }}\left(\pi^{\prime}\right) \leq \widehat{\pi}^{\prime}$.

(ii) $\alpha_{\mathrm{st}}\left(\sigma^{\prime}\right) \leq \widehat{\sigma}^{\prime}$. Since $\alpha_{\mathrm{va}}\left(b_{i}\right)=\widehat{b}_{i}$ and $\alpha_{\mathrm{val}}\left(d_{i}\right)=\widehat{d}_{i}$ for $1 \leq i \leq n$ Lemma 1 is applicable once more and gives us $\alpha_{\mathrm{st}}\left(\sigma^{\prime}\right) \leq \widehat{\sigma}^{\prime}$ and completes the proof of this case.

\section{Proof of Theorem 3}

Terminology. Analogously to our remark in section 6 on active components for rules AbsR in the abstract operational semantics it is possible to identify a similar pattern in the concrete operational semantics. Henceforth we will speak of the concrete active component $\left(\iota, q, q^{\prime}\right)$ of a rule $\mathbf{R}$ of the concrete operational semantics and we will say the abstract active component $\left(\widehat{\iota}, \widehat{q}, \widehat{q}^{\prime}\right)$ of a rule $\mathbf{A b s R}$ of the abstract operational semantics where AbsR is the abstract 
counterpart of $\mathbf{R}$. We will omit the adjectives abstract and concrete when there is no confusion.

Lemma 2. Suppose $s \rightarrow s^{\prime}$ using the concrete rule $\boldsymbol{R}$ with concrete active component $\left(\iota, q, q^{\prime}\right)$ and $\widehat{s} \geq \alpha_{c f a}(s)$. Then $\widehat{s} \rightsquigarrow \widehat{s}^{\prime}$ with $\widehat{s} \geq \alpha_{c f a}\left(s^{\prime}\right)$ using rule Abs $\boldsymbol{R}$ with abstract active component $\left(\alpha_{\text {pid }}(\iota), \alpha_{p s}(q), \alpha_{p s}\left(q^{\prime}\right)\right)$.

Proof. The claim follows from inspection of the proof of Theorem 1.

Proof of Theorem 3. Suppose $s \rightarrow s^{\prime}$ using rule $\mathbf{R}$ of the concrete operational semantics with active component $\left(\iota, q, q^{\prime}\right)$. We will prove our claim by case analysis on $\mathbf{R}$.

- $\mathbf{R}=$ FunEval, ArgEval, Apply or Vars. Take $\hat{s}=\alpha_{\mathrm{cfa}}(s)$; Lemma 2 gives us that $\widehat{s} \rightsquigarrow \widehat{s}^{\prime}$ using abstract rule AbsR = AbsFunEval, AbsArgEval, AbsApply or Vars respectively with active component $\left(\widehat{\iota}, \widehat{q}, \widehat{q}^{\prime}\right)$ where $\widehat{\iota}=\alpha_{\text {pid }}(\iota), \widehat{q}=\alpha_{\mathrm{ps}}(q)$ and $\widehat{q}^{\prime}=\alpha_{\mathrm{ps}}\left(q^{\prime}\right)$. It follows that

$$
\mathbf{r}:=\widehat{\iota}: \widehat{q} \stackrel{\tau}{\rightarrow} \widehat{q}^{\prime} \in R .
$$

Since $s \rightarrow s^{\prime}$ with active component $\left(\iota, q, q^{\prime}\right)$ it follows that (i) $\alpha_{\text {acs }}(s)(\widehat{\iota}, \widehat{q}) \geq 1$,

(ii) $\alpha_{\text {acs }}\left(s^{\prime}\right)(\widehat{\iota}, \widehat{q})=\alpha_{\text {acs }}(s)(\widehat{\iota}, \widehat{q})-1$ and

(iii) $\alpha_{\text {acs }}\left(s^{\prime}\right)\left(\widehat{\iota}, \widehat{q}^{\prime}\right)=\alpha_{\text {acs }}(s)\left(\widehat{\iota}, \widehat{q}^{\prime}\right)+1$

as $\widehat{\iota}=\alpha_{\text {pid }}(\iota), \widehat{q}=\alpha_{\mathrm{ps}}(q)$ and $\widehat{q}^{\prime}=\alpha_{\mathrm{ps}}\left(q^{\prime}\right)$. We know $\alpha_{\text {acs }}(s) \leq \mathbf{v}$ and thus

$$
\mathbf{v}(\widehat{\iota}, \widehat{q}) \geq 1
$$

If we define

$$
\mathbf{v}^{\prime}:=\mathbf{v}\left[(\widehat{\iota}, \widehat{q}) \mapsto \mathbf{v}(\widehat{\iota}, \widehat{q})-1,\left(\widehat{\iota}, \widehat{q}^{\prime}\right) \mapsto \mathbf{v}\left(\widehat{\iota}, \widehat{q}^{\prime}\right)+1\right],
$$

then it is clear that $\mathbf{v} \rightarrow_{\text {acs }} \mathbf{v}^{\prime}$ using rule $\mathbf{r} \in R$ and the inequalities

$$
\begin{gathered}
\alpha_{\mathrm{acs}}\left(s^{\prime}\right)(\widehat{\iota}, \widehat{q})=\alpha_{\mathrm{acs}}(s)(\widehat{\iota}, \widehat{q})-1 \leq \mathbf{v}(\widehat{\iota}, \widehat{q})-1=\mathbf{v}^{\prime}(\widehat{\iota}, \widehat{q}) \\
\alpha_{\mathrm{acs}}\left(s^{\prime}\right)\left(\widehat{\iota}, \widehat{q}^{\prime}\right)=\alpha_{\mathrm{acs}}(s)\left(\widehat{\iota}, \widehat{q}^{\prime}\right)+1 \leq \mathbf{v}\left(\widehat{\iota}, \widehat{q}^{\prime}\right)+1=\mathbf{v}^{\prime}\left(\widehat{\iota}, \widehat{q}^{\prime}\right) ;
\end{gathered}
$$

the consequence of the latter two is that $\alpha_{\text {acs }}\left(s^{\prime}\right) \leq \mathbf{v}^{\prime}$, since $\alpha_{\text {acs }}(s) \leq \mathbf{v}$, which completes the proof of this case.

- $\mathbf{R}=$ Receive. Letting $\widehat{s}=\alpha_{\text {cfa }}(s)$ Lemma 2 yields that $\widehat{s} \rightsquigarrow \widehat{s}^{\prime}$ using abstract rule AbsReceive with active component $\left(\widehat{\iota}, \widehat{q}, \widehat{q}^{\prime}\right)$ where $\widehat{\imath}=\alpha_{\text {pid }}(\iota), \widehat{q}=\alpha_{\mathrm{ps}}(q)$ and $\widehat{q}^{\prime}=\alpha_{\mathrm{ps}}\left(q^{\prime}\right)$. We note that $s=\langle\pi, \sigma, \mu\rangle$ and $\widehat{s}=\langle\widehat{\pi}, \widehat{\sigma}, \widehat{\mu}\rangle$ where $\widehat{\pi}=\alpha_{\text {proc }}(\pi), \widehat{\sigma}=$ $\alpha_{\mathrm{st}}(\sigma)$ and $\widehat{\mu}=\alpha_{\mathrm{ms}}(\mu)$. Let the message matched by mmatch and extracted from $\mu(\iota)$ be $d=\left(p_{i}, \rho^{\prime}\right)$ then inspecting rule AbsReceive we can assume that during $\widehat{s} \rightsquigarrow \widehat{s}^{\prime}$ message $\widehat{d}=$ $\left(p_{i}, \widehat{\rho}^{\prime}\right)$, where $\widehat{\rho}^{\prime}=\alpha_{\text {env }}\left(\rho^{\prime}\right)$, is matched by mmatch. Since the message abstraction is a sound data abstraction we know that

$$
\widehat{m}:=\alpha_{\mathrm{msg}}(\operatorname{res}(\sigma, d)) \in \widehat{\operatorname{res}}_{\mathrm{msg}}(\widehat{\sigma}, \widehat{d})
$$

and hence we have

$$
\mathbf{r}:=\widehat{\iota}: \widehat{q} \stackrel{?}{\longrightarrow} \widehat{q}^{\prime} \in R
$$

Additionally we know

(i) $\alpha_{\text {acs }}(s)(\widehat{\iota}, \widehat{q}) \geq 1$,

(ii) $\alpha_{\text {acs }}(s)(\widehat{\iota}, \widehat{m}) \geq 1$,

(iii) $\alpha_{\text {acs }}\left(s^{\prime}\right)(\widehat{\iota}, \widehat{q})=\alpha_{\text {acs }}(s)(\widehat{\iota}, \widehat{q})-1$,

(iv) $\alpha_{\text {acs }}\left(s^{\prime}\right)\left(\widehat{\iota}, \widehat{q}^{\prime}\right)=\alpha_{\text {acs }}(s)\left(\widehat{\iota}, \widehat{q}^{\prime}\right)+1$ and

(v) $\alpha_{\text {acs }}\left(s^{\prime}\right)(\widehat{\iota}, \widehat{m})=\alpha_{\text {acs }}(s)(\widehat{\iota}, \widehat{m})-1$

since $d$ is the message extracted from $\mu(\iota)$ and $\widehat{m}=\alpha_{\mathrm{msg}}(\operatorname{res}(\sigma, d))$. By assumption we know $\alpha_{\text {acs }}(s) \leq \mathbf{v}$ which implies

$$
\mathbf{v}(\widehat{\iota}, \widehat{q}) \geq 1 \text { and } \mathbf{v}(\widehat{\iota}, \widehat{m}) \geq 1
$$

and so we can define

$$
\mathbf{v}^{\prime}:=\mathbf{v}\left[\begin{array}{c}
(\widehat{\iota}, \widehat{q}) \mapsto \mathbf{v}(\widehat{\iota}, \widehat{q})-1, \\
(\widehat{\iota}, \widehat{m}) \mapsto \mathbf{v}(\widehat{\iota}, \widehat{m})-1, \\
\left(\widehat{\iota}, \widehat{q}^{\prime}\right) \mapsto \mathbf{v}\left(\widehat{\iota}, \widehat{q}^{\prime}\right)+1
\end{array}\right] ;
$$

it is then clear that, using rule $\mathbf{r} \in R, \mathbf{v} \rightarrow$ acs $\mathbf{v}^{\prime}$ and

$$
\begin{gathered}
\alpha_{\text {acs }}\left(s^{\prime}\right)(\widehat{\iota}, \widehat{q})=\alpha_{\text {acs }}(s)(\widehat{\iota}, \widehat{q})-1 \leq \mathbf{v}(\widehat{\iota}, \widehat{q})-1=\mathbf{v}^{\prime}(\widehat{\iota}, \widehat{q}) \\
\alpha_{\text {acs }}\left(s^{\prime}\right)(\widehat{\iota}, \widehat{m})=\alpha_{\text {acs }}(s)(\widehat{\iota}, \widehat{m})-1 \leq \mathbf{v}(\widehat{\iota}, \widehat{m})-1=\mathbf{v}^{\prime}(\widehat{\iota}, \widehat{m}) \\
\alpha_{\text {acs }}\left(s^{\prime}\right)\left(\widehat{\iota}, \widehat{q}^{\prime}\right)=\alpha_{\text {acs }}(s)\left(\widehat{\iota}, \widehat{q}^{\prime}\right)+1 \leq \mathbf{v}\left(\widehat{\iota}, \widehat{q}^{\prime}\right)+1=\mathbf{v}^{\prime}\left(\widehat{\iota}, \widehat{q}^{\prime}\right) .
\end{gathered}
$$

Hence, since $\alpha_{\text {acs }}(s) \leq \mathbf{v}$, we can conclude $\alpha_{\text {acs }}\left(s^{\prime}\right) \leq \mathbf{v}^{\prime}$ as desired.

- $\mathbf{R}=$ Send. Using Lemma 2 , with $\widehat{s}=\alpha_{\mathrm{cfa}}(s)$, gives $\widehat{s} \rightsquigarrow \widehat{s}^{\prime}$ with active component $\left(\widehat{\iota}, \widehat{q}, \widehat{q}^{\prime}\right)$ for the abstract rule AbsSend where $\widehat{\iota}=\alpha_{\text {pid }}(\iota), \widehat{q}=\alpha_{\mathrm{ps}}(q)$ and $\widehat{q}^{\prime}=\alpha_{\mathrm{ps}}\left(q^{\prime}\right)$. Examining the concrete and abstract states we see $s=\langle\pi, \sigma, \mu\rangle$ and $\widehat{s}=\langle\widehat{\pi}$, $\widehat{\sigma}, \widehat{\mu}\rangle$ where $\widehat{\pi}=\alpha_{\text {proc }}(\pi), \widehat{\sigma}=\alpha_{\text {st }}(\sigma)$ and $\widehat{\mu}=\alpha_{\text {ms }}(\mu)$. Let the pid of the recipient be $\iota^{\prime}$ and let $d$ be the value enqueued to $\iota^{\prime}$ 's mailbox $\mu\left(\iota^{\prime}\right)$; inspecting the proof of Theorem 1 the pid of the abstract recipient is $\widehat{\iota}:=\alpha_{\text {pid }}\left(\iota^{\prime}\right)$ and the sent abstract value is $\widehat{d}=\alpha_{\text {val }}(d)$. Appealing to the soundness of the message abstraction we obtain

$$
\widehat{m}:=\alpha_{\mathrm{msg}}(\operatorname{res}(\sigma, d)) \in \widehat{\operatorname{res}}_{\mathrm{msg}}(\widehat{\sigma}, \widehat{d})
$$

and hence we have

$$
\mathbf{r}:=\widehat{\iota}: \widehat{q} \stackrel{\widehat{\iota}^{\prime} ! \widehat{m}}{\longrightarrow} \widehat{q}^{\prime} \in R
$$

Additionally we know

(i) $\alpha_{\text {acs }}(s)(\widehat{\iota}, \widehat{q}) \geq 1$,

(ii) $\alpha_{\text {acs }}\left(s^{\prime}\right)(\widehat{\iota}, \widehat{q})=\alpha_{\text {acs }}(s)(\widehat{\iota}, \widehat{q})-1$,

(iii) $\alpha_{\text {acs }}\left(s^{\prime}\right)\left(\widehat{\iota}, \widehat{q}^{\prime}\right)=\alpha_{\text {acs }}(s)\left(\widehat{\iota}, \widehat{q}^{\prime}\right)+1$ and

(iv) $\alpha_{\text {acs }}\left(s^{\prime}\right)(\widehat{\iota}, \widehat{m})=\alpha_{\text {acs }}(s)(\widehat{\iota}, \widehat{m})+1$

since $d$ is the message enqueued to $\mu\left(\iota^{\prime}\right)$ and $\widehat{m}=\alpha_{\operatorname{msg}}(\operatorname{res}(\sigma, d))$. From our assumption we know $\alpha_{\text {acs }}(s) \leq \mathbf{v}$ and thus

$$
\mathbf{v}(\widehat{\iota}, \widehat{q}) \geq 1
$$

making the definition

$$
\mathbf{v}^{\prime}:=\mathbf{v}\left[\begin{array}{rl}
(\widehat{\iota}, \widehat{q}) & \mapsto \mathbf{v}(\widehat{\iota}, \widehat{q})-1, \\
\left(\widehat{\iota}, \widehat{q}^{\prime}\right) & \mapsto \mathbf{v}\left(\widehat{\iota}, \widehat{q}^{\prime}\right)+1, \\
(\widehat{\iota}, \widehat{m}) & \mapsto \mathbf{v}(\widehat{\iota}, \widehat{m})+1
\end{array}\right] ;
$$

we observe that we are able to use rule $\mathbf{r} \in R$ to make the step $\mathbf{v} \rightarrow$ acs $\mathbf{v}^{\prime}$. Further the inequalities

$$
\begin{gathered}
\alpha_{\text {acs }}\left(s^{\prime}\right)(\widehat{\iota}, \widehat{q})=\alpha_{\text {acs }}(s)(\widehat{\iota}, \widehat{q})-1 \leq \mathbf{v}(\widehat{\iota}, \widehat{q})-1=\mathbf{v}^{\prime}(\widehat{\iota}, \widehat{q}) \\
\alpha_{\text {acs }}\left(s^{\prime}\right)\left(\widehat{\iota}, \widehat{q}^{\prime}\right)=\alpha_{\text {acs }}(s)\left(\widehat{\iota}, \widehat{q}^{\prime}\right)+1 \leq \mathbf{v}\left(\widehat{\iota}, \widehat{q}^{\prime}\right)+1=\mathbf{v}^{\prime}\left(\widehat{\iota}, \widehat{q}^{\prime}\right) \\
\alpha_{\text {acs }}\left(s^{\prime}\right)(\widehat{\iota}, \widehat{m})=\alpha_{\text {acs }}(s)(\widehat{\iota}, \widehat{m})-1 \leq \mathbf{v}(\widehat{\iota}, \widehat{m})-1=\mathbf{v}^{\prime}(\widehat{\iota}, \widehat{m}) .
\end{gathered}
$$

imply, since $\alpha_{\text {acs }}(s) \leq \mathbf{v}$, that $\alpha_{\text {acs }}\left(s^{\prime}\right) \leq \mathbf{v}^{\prime}$ which concludes the proof of this case.

- $\mathbf{R}=$ Spawn. Take $\widehat{s}=\alpha_{\mathrm{cfa}}(s)$; Lemma 2 gives us that $\widehat{s} \rightsquigarrow \widehat{s}$ using abstract rule AbsSpawn with active component $\left(\widehat{\iota}, \widehat{q}, \widehat{q}^{\prime}\right)$ where $\widehat{\iota}=\alpha_{\text {pid }}(\iota), \widehat{q}=\alpha_{\mathrm{ps}}(q)$ and $\widehat{q}^{\prime}=\alpha_{\mathrm{ps}}\left(q^{\prime}\right)$. We note that $s=\langle\pi, \sigma, \mu\rangle, s^{\prime}=\left\langle\pi^{\prime}, \sigma^{\prime}, \mu^{\prime}\right\rangle$ and $\widehat{s}=\langle\widehat{\pi}, \widehat{\sigma}, \widehat{\mu}\rangle$ where $\widehat{\pi}=\alpha_{\text {proc }}(\pi), \widehat{\sigma}=\alpha_{\text {st }}(\sigma)$ and $\widehat{\mu}=\alpha_{\mathrm{ms}}(\mu)$. Further we can assume

$$
\begin{aligned}
\pi(\iota) & =\langle\operatorname{fun}() \rightarrow e, \rho, a, t\rangle \\
\sigma(a) & =\operatorname{Arg}_{1}\left\langle\ell, d, \rho^{\prime}, c\right\rangle \\
d & =\left(\mathbf{s p a w n}_{-}\right) \\
\iota^{\prime} & :=\operatorname{new}_{\text {pid }}(\iota, \ell, \vartheta)
\end{aligned}
$$




$$
\begin{aligned}
\pi^{\prime}(\iota) & =\left\langle\iota^{\prime}, \rho^{\prime}, c, t\right\rangle=q^{\prime} \\
\pi^{\prime}\left(\iota^{\prime}\right) & =\left\langle e, \rho, *, t_{0}\right\rangle=: q^{\prime \prime}
\end{aligned}
$$

Noting that we are replicating the step $s \rightarrow s^{\prime}$ in the abstract $\widehat{s} \rightsquigarrow \widehat{s}^{\prime}, \alpha_{\text {cfa }}(s)=\widehat{s}$ and $\alpha_{\text {pid }} \circ$ new pid $=\widehat{\text { newpid }} \circ \alpha$ we can see that the new abstract pid created is $\widehat{\iota}=\alpha_{\text {pid }}\left(\iota^{\prime}\right)$ together with its process state $\widehat{q}^{\prime \prime}=\alpha_{\mathrm{ps}}\left(q^{\prime \prime}\right)$. Hence we can conclude that

$$
\mathbf{r}:=\widehat{\iota}: \widehat{q} \stackrel{\nu \widehat{\iota}^{\prime} \cdot \widehat{q}^{\prime \prime}}{\longrightarrow} \widehat{q}^{\prime} \in R
$$

and we observe that

(i) $\alpha_{\text {acs }}(s)(\widehat{\iota}, \widehat{q}) \geq 1$,

(ii) $\alpha_{\text {acs }}\left(s^{\prime}\right)(\widehat{\iota}, \widehat{q})=\alpha_{\text {acs }}(s)(\widehat{\iota}, \widehat{q})-1$,

(iii) $\alpha_{\text {acs }}\left(s^{\prime}\right)\left(\widehat{\iota}, \widehat{q}^{\prime}\right)=\alpha_{\text {acs }}(s)\left(\widehat{\iota}, \widehat{q}^{\prime}\right)+1$ and

(iv) $\alpha_{\text {acs }}\left(s^{\prime}\right)\left(\widehat{\iota}^{\prime}, \widehat{q}^{\prime \prime}\right)=\alpha_{\text {acs }}(s)\left(\widehat{\iota}, \widehat{q}^{\prime \prime}\right)+1$.

Now the assumption $\alpha_{\text {acs }}(s) \leq \mathbf{v}$ allows us to conclude

$$
\mathbf{v}(\widehat{\iota}, \widehat{q}) \geq 1
$$

so that we can define

$$
\mathbf{v}^{\prime}:=\mathbf{v}\left[\begin{array}{rl}
(\widehat{\iota}, \widehat{q}) & \mapsto \mathbf{v}(\widehat{\iota}, \widehat{q})-1, \\
\left(\widehat{\iota}, \widehat{q}^{\prime}\right) & \mapsto \mathbf{v}\left(\widehat{\iota}, \widehat{q}^{\prime}\right)+1, \\
\left(\widehat{\iota}, \widehat{q}^{\prime \prime}\right) & \mapsto \mathbf{v}\left(\widehat{\iota}, \widehat{q}^{\prime \prime}\right)+1
\end{array}\right] ;
$$

and use rule $\mathbf{r} \in R$ to make the step $\mathbf{v} \rightarrow$ acs $\mathbf{v}^{\prime}$. Further with the inequalities

$$
\begin{gathered}
\alpha_{\text {acs }}\left(s^{\prime}\right)(\widehat{\iota}, \widehat{q})=\alpha_{\text {acs }}(s)(\widehat{\iota}, \widehat{q})-1 \leq \mathbf{v}(\widehat{\iota}, \widehat{q})-1=\mathbf{v}^{\prime}(\widehat{\iota}, \widehat{q}) \\
\alpha_{\text {acs }}\left(s^{\prime}\right)\left(\widehat{\iota}, \widehat{q}^{\prime}\right)=\alpha_{\text {acs }}(s)\left(\widehat{\iota}, \widehat{q}^{\prime}\right)+1 \leq \mathbf{v}\left(\widehat{\iota}, \widehat{q}^{\prime}\right)+1=\mathbf{v}^{\prime}\left(\widehat{\iota}, \widehat{q}^{\prime}\right) \\
\alpha_{\text {acs }}\left(s^{\prime}\right)\left(\widehat{\iota}^{\prime}, \widehat{q}^{\prime \prime}\right)=\alpha_{\text {acs }}(s)\left(\widehat{\iota}^{\prime}, \widehat{q}^{\prime \prime}\right)-1 \leq \mathbf{v}\left(\widehat{\iota}, \widehat{q}^{\prime \prime}\right)-1=\mathbf{v}^{\prime}\left(\widehat{\iota}, \widehat{q}^{\prime \prime}\right) .
\end{gathered}
$$

and our assumption $\alpha_{\text {acs }}(s) \leq \mathbf{v}$ we see that $\alpha_{\text {acs }}\left(s^{\prime}\right) \leq \mathbf{v}^{\prime}$ which completes the proof of this case and the theorem. 